\title{
ARTICLE OPEN Predicting surface deformation during mechanical attrition of metallic alloys
}

\author{
Shan Cecilia Cao ${ }^{1}$, Xiaochun Zhang ${ }^{2}$, Jian Lu ${ }^{3}{ }^{3}$, Yongli Wang ${ }^{3,4}$, San-Qiang Shi ${ }^{4}$ and Robert O. Ritchie (iD) ${ }^{1,5}$
}

Extensive efforts have been devoted in both the engineering and scientific domains to seek new designs and processing techniques capable of making stronger and tougher materials. One such method for enhancing such damage-tolerance in metallic alloys is a surface nano-crystallization technology that involves the use of hundreds of small hard balls which are vibrated using high-power ultrasound so that they impact onto the surface of a material at high speed (termed Surface Mechanical Attrition Treatment or SMAT). However, few studies have been devoted to the precise underlying mechanical mechanisms associated with this technology and the effect of processing parameters. As SMAT is dynamic plastic deformation process, here we use random impact deformation as a means to investigate the relationship between impact deformation and the parameters involved in the processing, specifically ball size, impact velocity, ball density and kinetic energy. Using analytical and numerical solutions, we examine the size of the indents and the depths of the associated plastic zones induced by random impacts, with results verified by experiment in austenitic stainless steels. In addition, global random impact and local impact frequency models are developed to analyze the statistical characteristics of random impact coverage, together with a description of the effect of random multiple impacts, which are more reflective of SMAT. We believe that these models will serve as a necessary foundation for further, and more energy-efficient, development of such surface nano-crystalline processing technologies for the strengthening of metallic materials.

npj Computational Materials (2019)5:36; https://doi.org/10.1038/s41524-019-0171-6

\section{INTRODUCTION}

Deformation processes based on the impact of particles have been utilized extensively to harden surfaces, by imparting residual compressive stresses and/or by creating a work-hardened layer for the purpose of improving surface properties, such as resistance to fatigue or wear. ${ }^{1,2}$ More recently, these techniques have also been used to make stronger engineering metal alloys, ${ }^{3-5}$ through the use of techniques such as air blast and ultrasonic shot peening, ${ }^{6}$ surface mechanical attrition treatment, ${ }^{7}$ particle impact processing, ${ }^{8}$ and surface nano-crystallization and hardening, ${ }^{9}$ specifically to develop surface hardness and structural gradients. Here we focus on the technique of surface mechanical attrition treatment (or SMAT) which has been widely used in the generation of nanostructures and subsurface gradients to improve the damagetolerance of structural materials. ${ }^{10-13}$ The technique was first introduced by $\mathrm{Lu}$ and $\mathrm{Lu}^{14}$ who exploited an ultrasonic set-up to vibrate spherical balls actuated by high-power ultrasound. ${ }^{15}$ During this process, hard spherical balls are randomly impacted on surface of target materials, specifically to establish nano-sized crystalline layers on a variety of metallic materials, ${ }^{16-20}$ including steels, nickel-based alloys and Mg-based alloys. ${ }^{21}$ However, as numerous indents are generated on the sample surface layer by such impacts, the successful use of SMAT requires a number of parameters to be effectively controlled, including ball size, ball density, impact velocity and impact kinetic energy.

One good example of the role of SMAT in enhancing the properties of metallic alloys is illustrated in Fig. 1 for an AISI $316 \mathrm{~L}$ austenitic stainless steel, where we show how the process can induce superior mechanical properties through surface modification. ${ }^{3,4}$ Specifically, we compare the microstructure and the mechanical behavior of a SMAT-treated AISI $316 \mathrm{~L}$ stainless steel with that of an as-annealed AISI $316 \mathrm{~L}$ stainless steel. Following SMAT treatments, the hardness distribution along the crosssection of the SMAT-treated steel is a factor of $\sim 3-4$ times higher than that of the as-annealed steel (Fig. 1a), which is consistent with the uniaxial tensile test results (for both engineering and true stress/strains) which show corresponding factors of 4- and 3-fold increases in the yield and tensile strengths, respectively (Fig. 1b). Cross-section scanning electron microscopy (SEM) and electron backscatter diffraction (EBSD) images and related schematic illustrations show that this results from a gradient microstructure in grain size in the SMAT-treated steel from surface to core (from $\sim 80 \mathrm{~nm}$ to $\sim 20 \mu \mathrm{m}$ ), with dual-phase crystal phases comprising alternating nano-band austenite and nano-lamellar martensite within each grain ${ }^{3,4}$ (Fig. 1c, e, g), as compared to a nominally equiaxed single-phase austenitic grain structure in the annealed steel (Fig. 1d, f, h). A comparison in Fig. 1i, j with other highstrength steels, namely dual-phase steels and TRIP-steels, ${ }^{22,23}$ of the tensile and yield strength properties of the SMAT-treated stainless steel as a function of their tensile ductility, clearly reveals that SMAT treatments have the capacity to radically enhance the damage-tolerant properties of medium- to high-strength steels in terms of much improved combinations of strength and ductility.

The purpose of the current work is to provide a relaible mechanistic and modeling basis for the successful adoption of

\footnotetext{
${ }^{1}$ Department of Materials Science \& Engineering, University of California, Berkeley, CA 94720, USA; ${ }^{2}$ Shanghai Institute of Applied Physics, Chinese Academy of Sciences, Shanghai 201800, China; ${ }^{3}$ Department of Mechanical Engineering, City University of Hong Kong, Hong Kong, China; ${ }^{4}$ Department of Mechanical Engineering, The Hong Kong Polytechnic University, Hung Hom, Hong Kong and ${ }^{5}$ Materials Sciences Division, Lawrence Berkeley National Laboratory, Berkeley, CA 94720, USA

Correspondence: Xiaochun Zhang (zhangxiaochun@sinap.ac.cn) or Robert O. Ritchie (roritchie@lbl.gov)
}

Received: 10 August 2018 Accepted: 15 February 2019

Published online: 15 March 2019 
surface nano-crystallization for the purpose of inducing these exceptional mechanical properties. Specifically, we use analytical modeling and numerical analysis of the indent size induced by random impacts, as a function of the parameters such as the ball size, ball density, impact velocity and kinetic energy, and of the resulting depths of the impact plastic zones; theoretical results are then validated by experiment. Based on this, a global random impact model and a local impact frequency model are developed to analyze the statistical characteristics of the impact coverage on the material under treatment. We further provide an analogous model to describe the phenomenon of full coverage by random multiple impacts, which is most representative of SMAT. Given

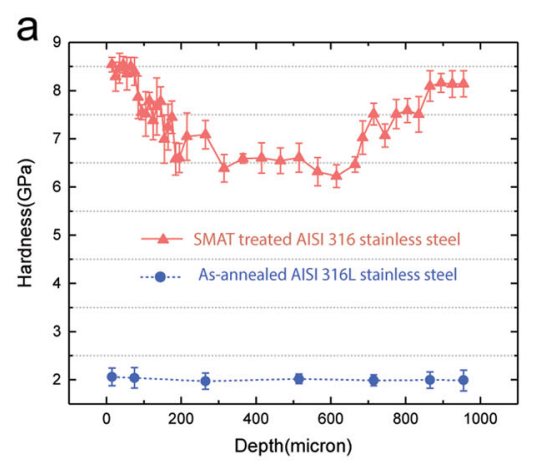

C

SMAT treated AISI 316 stainless steel b

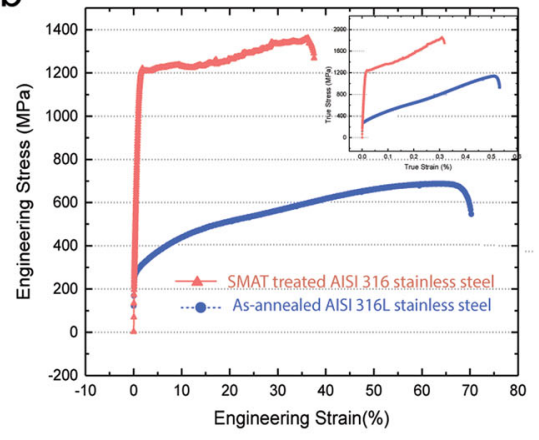

d

As-annealed AISI 316L stainless steel

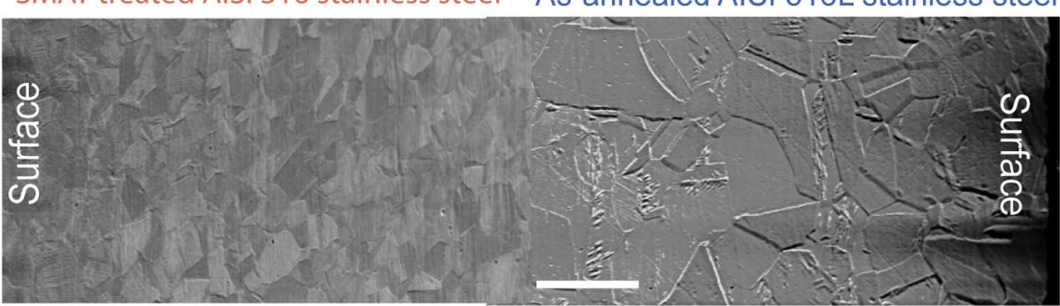

e

f

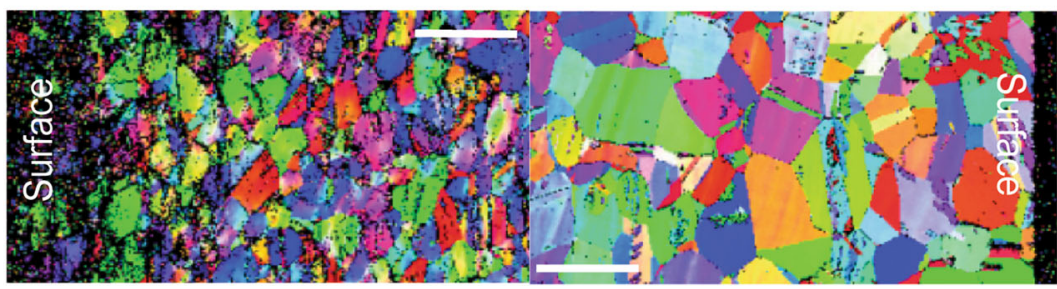

g

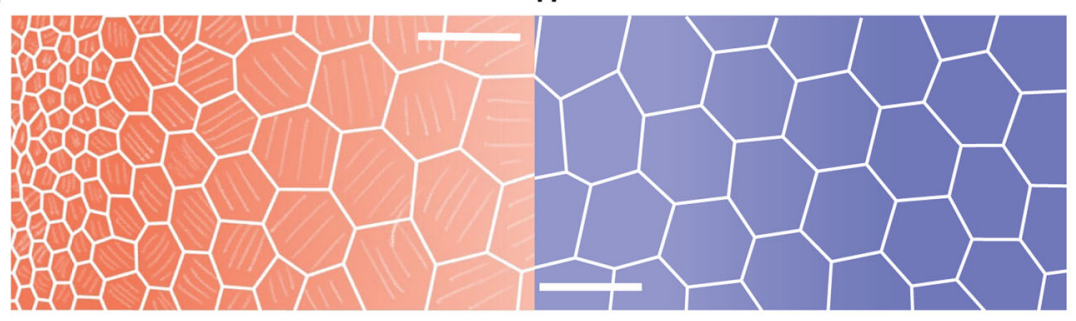

Surface

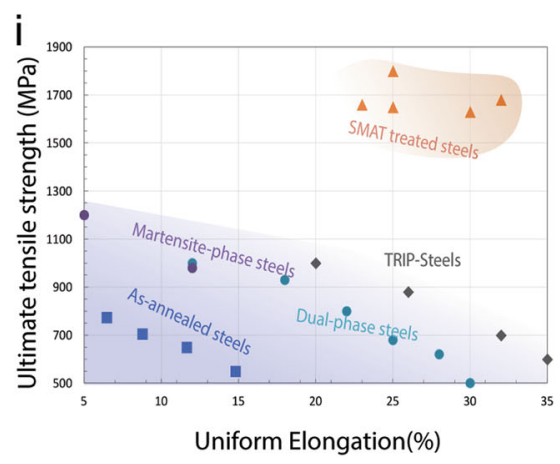

$\mathrm{h}$

Core

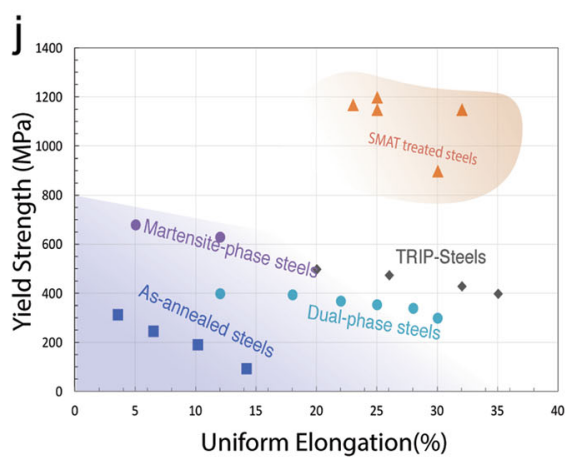


Fig. 1 Mechanical performance and microstructure comparation of SMAT-treated and as-annealed AISI 316L stainless steel: a Hardness distribution along the cross-section of an as-annealed AISI 316 L stainless steel, as compared to SMAT-treated AISI $316 \mathrm{~L}$ stainless steel. b Uniaxial tensile tests show engineering stress-strain curves of the SMAT-treated AISI 316 L stainless steel, as compared with that of asannealed AISI 316 L stainless steel; inset graphs show the corresponding true stress-strain curves. Cross-sectional SEM images of c SMATtreated AISI $316 \mathrm{~L}$ stainless steel and d as-annealed AISI $316 \mathrm{~L}$ stainless steel, and corresponding cross-sectional EBSD images, respectively, in e, f AISI $316 \mathrm{~L}$ stainless steel, schematic drawing for both steels in $\mathbf{g}, \mathbf{h}$. The striped regions inside each grain shown in the SEM images represents the nano-dual-phase morphology ${ }^{4}$ found in the treated steels. (Scale bars for $\mathbf{c}$ through $\mathbf{h}$ represent $100 \mu \mathrm{m}$ ). $\mathbf{i}$, $\mathbf{j}$ Representative tensile properties of steels. Red triangle data points represent the tensile properties of SMAT-treated steels. ${ }^{22} \mathrm{~A}$ comparison of the respective $\mathbf{i}$ yield and $\mathbf{j}$ ultimate tensile strengths of dual-phase steels, TRIP-steels, martensite-phase steels and SMAT-treated steels, indicates that the SMATtreated steels have a far improved combination of strength and ductility in comparison to other steels

that the salient mechanisms responsible for the nano-structuring are associated with surface nano-recrystallization induced by the plastic deformation and residual stresses associated with the impact events, we believe that our numerical and experimental observations, coupled with systematically developed modeling, can afford the necessary foundation for further development and optimization of superior surface mechanical attrition procedures.

The SMAT treatment process is a dynamic plastic deformation process, where plates of the material to be treated are placed on the top side of a chamber containing hundreds of 3-mm diameter hard balls, which are vibrated using high-power ultrasound so that they impact onto the plate surfaces at high speed. Plastic deformation in the surface layers, coupled with the large strains and high strain rates, result in a progressive reduction of the surface microstructure into nanograins. Indentation size is important here to achieving $100 \%$ coverage; moreover, the depth of plastic zone beneath the intents is also critical as it determines the thickness of the work-hardened surface layer as well as depth below the surface where beneficial residual compressive stresses are created. To fully understand and optimize the SMAT process, in current study quantitative relationships between SMAT parameters, indent size and plastic-zone size are determined and validated by experiment.

Previous studies ${ }^{24}$ on WC/Co ball impacts on Ni-base C-2000 Hastelloy plate revealed that the plastic-zone depth increases with the kinetic energy of balls and, for a given kinetic energy, the ball size. Associated analytical models ${ }^{25,26}$ to predict the surface deformation in a component induced by high-energy impacts have been mainly based on Hertzian theory for the elastic contact between a sphere and a semi-infinite solid, ${ }^{27}$ where depth of the plastic zone $\left(r_{\mathrm{y}}\right)$ induced by high-energy ball impacts has the following dependencies on the density $\rho_{\mathrm{B}}$, normal impact velocity $v_{\mathrm{n}}$ and diameter $d_{\mathrm{B}}$ of the impacting ball: ${ }^{24}$

$r_{\mathrm{y}} \propto \rho_{\mathrm{B}}^{1 / 5}, r_{\mathrm{y}} \propto v_{\mathrm{n}}^{3 / 20}, r_{\mathrm{y}} \propto d_{\mathrm{B}}^{2 / 3}$.

These analytical approaches though neglect factors such as work hardening, strain-rate sensitivity, friction and thermal effects. The work of Dai ${ }^{31,32}$ offers an analytical model to predict the surface roughness (indent depth) of aluminum 5052 plates treated with a surface nano-crystallization and hardening process, but this specific approach ${ }^{32}$ only takes into consideration the strainhardening effect during the impact process and merely provides the means to calculate the peak-to-valley size of the impactdamaged zones. Moreover, Dai's analyses are only strictly applicable to deformation induced by normal impacts, i.e., perpendicular to the surface of the target solid. During SMAT, the impact processes are random with a high strain rate under impact deformation. Accordingly, to accurately predict the response of a sample submitted to such surface treatments, the effects of high strain rates on the flow stress must be included in the constitutive models. To address this, we apply here the Johnson-Cook (J-C) plasticity hardening model ${ }^{28}$ to estimate the plastic zones induced by high strain-rate random impacts involving elastic-plastic dynamic deformation. This model represents an empirical constitutive equation that was developed from the experimental study of the fracture characteristics of metals subjected to various strains, strain rates, temperatures and pressures. $^{28}$ The equivalent flow stress, $\sigma_{f}$, in J-C model is given by:

$$
\sigma_{f}=\left[\sigma_{y}+B(\bar{\varepsilon})^{n}\right]\left[1+C \ln \left(\frac{\dot{\bar{\varepsilon}}}{\dot{\varepsilon}_{0}}\right)\right]\left[1-\left(\frac{T-T_{\mathrm{r}}}{T_{\mathrm{m}}-T_{\mathrm{r}}}\right)^{\mathrm{m}}\right],
$$

where $\sigma_{y}$ is the initial yield strength of the material at reference temperature $\left(T_{\mathrm{r}}\right), \bar{\varepsilon}$ is the equivalent strain, and $B, n, C$ and $m$ are material constants. Specifically, the constant $B$ and strainhardening exponent $n$ represent the constitutive law at low homologous temperatures, $C$ and $m$ are the corresponding ratedependent constants which respectively represent the coefficient of strain-rate hardening and the thermal-softening exponent, $T$ and $T_{\mathrm{m}}$ are respectively the current and material's melting temperature, and $\dot{\bar{\varepsilon}}$ is the equivalent plastic strain rate which is normalized in Eq. (2) by a reference strain rate $\dot{\varepsilon}_{0}$ set to unity.

In addition, we used finite element analysis (FEA), which can provide a powerful method for simulating single and multiple ball impacts onto a target, as the dynamic, high-velocity impact of a ball and the resulting elastic-plastic behavior of the target can be both readily analyzed. Figure $2 a, b$ illustrates the FEA model for the three-dimensional (3-D) dynamic analysis that was performed in this study using the commercial code Abaqus/Explicit mode. Eight-node linear brick elements with reduced integration (C3D8R) were used with the mesh modeled to be very fine close to the impact zone, to achieve good resolution of the plastic strain distribution beneath the surface, and to be coarser away from the impact zone. The ball was also fine meshed to ensure that it possessed a smooth spherial surface condition on impact during the SMAT process. Specifically, we used 194, 840 elements in the single impact model. The size of elements in the impact zone was chosen to be $1 / 24^{\text {th }}$ of dimple size; this is considerably smaller than previous mesh convergence studies ${ }^{33-35}$, which utilized elements that were $1 / 15^{\text {th }}$ and $1 / 10^{\text {th }}$ of the dimple size. The implication of this is that we have taken a smaller mesh size parameter than all previous methods to ensure convergence of our calculations. The target material plate was restrained against all displacements and rotations on the top end.

In this work, a widely-used AISI $316 \mathrm{~L}$ austenitic stainless steel was chosen as the target material; its nominal chemical composition and material properties (for the J-C model) are given, respectively, in Tables 1 and $2{ }^{29}$ Our experimental studies ${ }^{3,4,30}$ showed that both the hardness and yield/tensile strengths can be significantly improved in SMAT-treated materials (Fig. 1). Our numerical study is consistent with the experimental results; details are presented below.

\section{RESULTS}

Numerical analysis

To explore the salient micro-mechanisms which underpin these nano-structuring procedures, we designed the numerical simulation system depicted in Fig. 2. Schematic illustrations of the indentation process are shown in Fig. $2 c$, together with simulated 
a

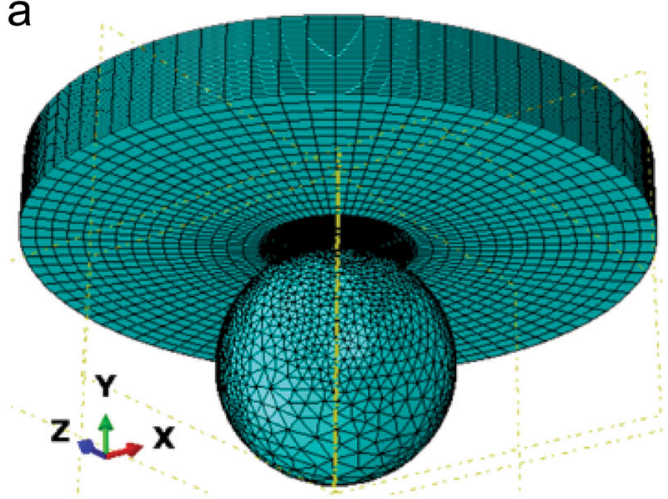

C

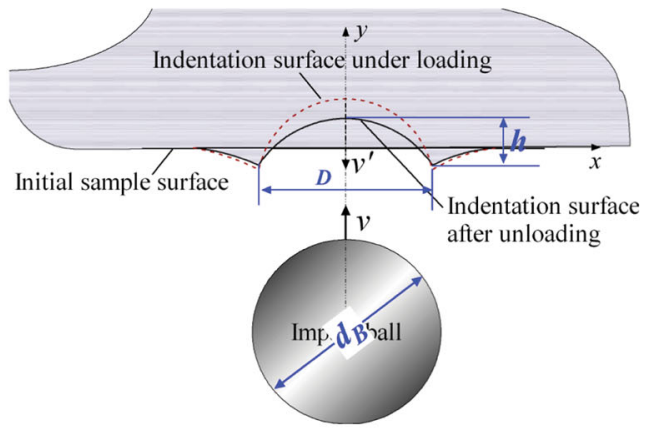

e
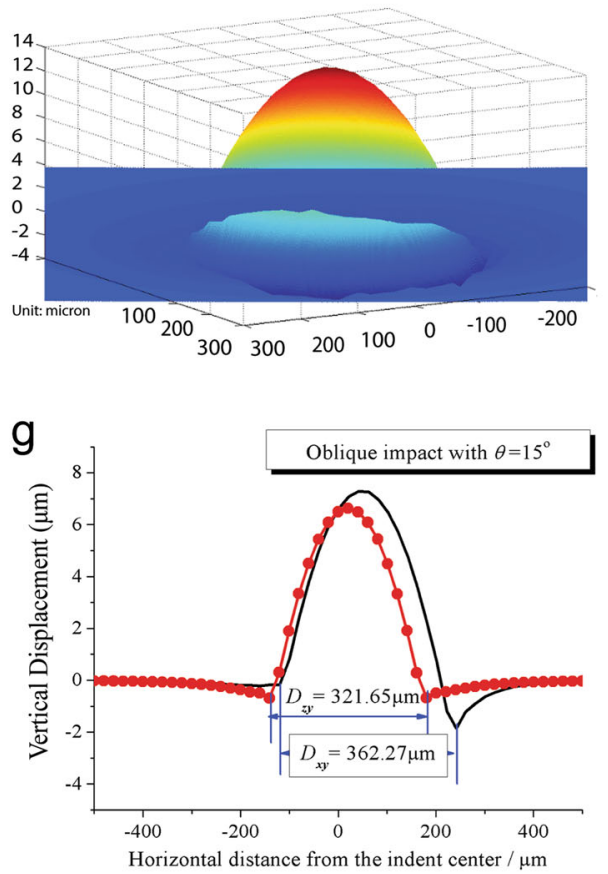

b

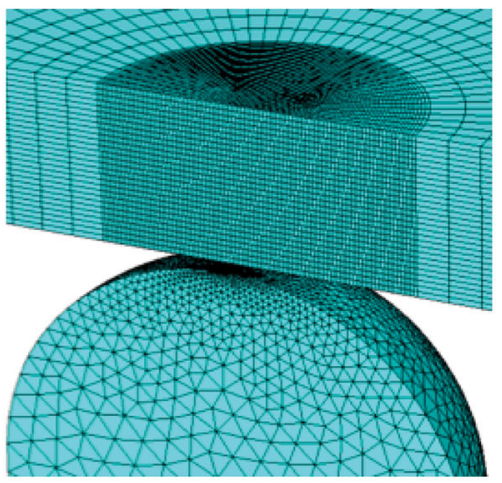

d

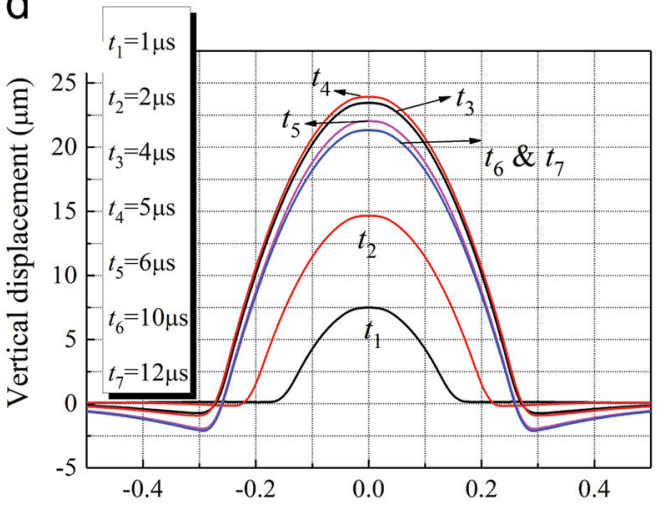

Horizontal distance from the indent center ( $\mathrm{mm}$ )

f

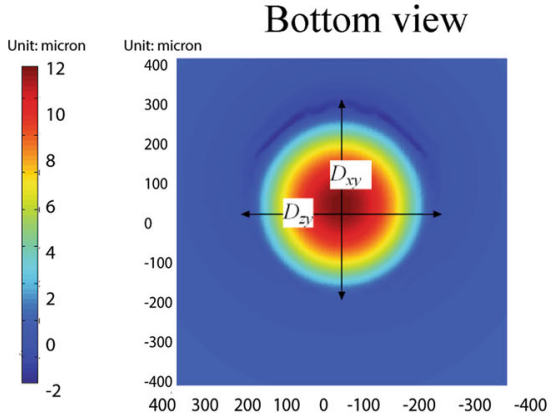

h

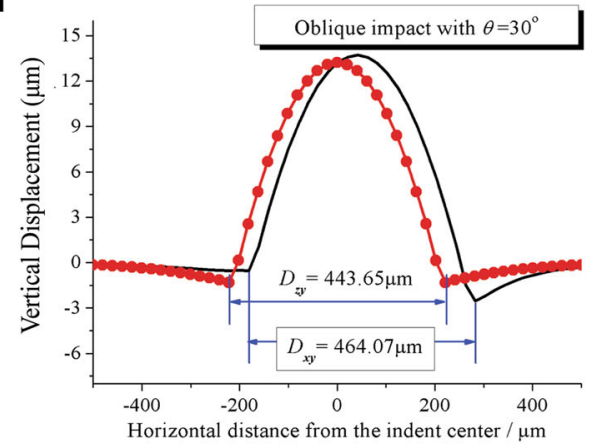

Fig. 2 Numerical simulation of indent size: FEA model for 3-D dynamic analysis to investigate the ball impact process shown in a as a full model view and in b. as a half model view. c Schematic illustration of one vertical impact process and $\mathbf{d}$ simulated contours of the indent generated by one 3-mm rigid ball with a velocity of $8 \mathrm{~m} / \mathrm{s}$. e, f 3-D contour plots of indentation surface generated by impact with oblique angle shown as e lateral view and $\mathbf{f}$ bottom view; false colors indicate displacements in micrometers. The indent shape is elliptical if impact angle $\theta$ is less than $90^{\circ}$. The contours of indent and the indent diameters along two crossover directions $D_{x y}$ and $D_{z y}$ are measured for the impact cases $\mathbf{g}$ at $\theta=15^{\circ}$, and $\mathbf{h}$ at $\theta=30^{\circ}$ 
Table 1. Chemical composition of AISI $316 \mathrm{~L}$

\begin{tabular}{ll}
\hline Element & Wt.\% \\
\hline Iron & $62-72$ \\
Chromium & $16-18$ \\
Nickel & $10-14$ \\
Molybdenum & $2-3$ \\
Manganese & 2.0 \\
Sulfur & 0.03 \\
Carbon & 0.03 \\
Silicon & 1.0 \\
Phosphorous & 0.045
\end{tabular}

Table 2. Values of parameters used in the J-C model (Eq. (2)) for AISI $316 \mathrm{~L}$ stainless steel

\begin{tabular}{ll}
\hline Material property & Value used for AISI $316 \mathrm{~L}$ \\
\hline Density $\left(\mathrm{kg} / \mathrm{m}^{3}\right)$ & 8,000 \\
Young's modulus, $E(\mathrm{GPa})$ & 193 \\
Poisson's ratio, $\nu$ & 0.3 \\
Initial yield strength, $\sigma_{\mathrm{y}}(\mathrm{MPa})$ & 305 \\
$B(\mathrm{MPa})$ & 1161 \\
$n$ & 0.61 \\
$C$ & 0.01 \\
$m$ & 0.52 \\
\hline
\end{tabular}

contours of the indent created by one impact of a rigid ball (Fig. $2 \mathrm{~d}) ; d_{\mathrm{B}}$ is the ball diameter, $v$ and $v^{\prime}$ are, respectively, the impingement and rebound velocities, $D$ is the indent diameter and $h$ is the indent depth. For a $3-\mathrm{mm}$ ball impacting on an AISI $316 \mathrm{~L}$ stainless steel surface at a speed of $8 \mathrm{~m} / \mathrm{s}$, the depth of the indent increases with change in time from $t=0$ to $\sim 5 \mu \mathrm{s}$, whereupon the depth subsequently is reduced due to springback (Fig. 2d). In addition, a small pile-up is generated near the edge of indent. The impact angle $\theta$, defined as the angle between the impact direction and the horizontal plane in 3-D space, has an important effect on the indent size. Indeed, the indent shape will be elliptical if $\theta$ is less than $90^{\circ}$ (Fig. 2e, f). The indent diameters along two crossover directions, $D_{x y}$ and $D_{z y}$, are measured for the impacts at $\theta=15^{\circ}$ and $\theta=30^{\circ}$, respectively (Fig. $2 \mathrm{~g}, \mathrm{~h}$ ). As the ellipticities, $D_{x y} / D_{z y}$, are not large, for simplification the indent shape can be regarded as being circular in our study. Note that the model developed here is a macroscopic model without emphasis on nano-/micro-scale phenomena such as dislocation activity. However, we consider the subsequent recovery following impact, as shown in Fig. 2d; after the ball bounces back off the surface, a permanent indent is generated, the final indent depth of which is determined by the plastic deformation during contact and elastic recovery from spring-back to an equilibrium position where all internal stresses are relatively in balance.

Numerical solutions for indent size under normal impact

The indent size generated by normal impacts is significantly influenced by the ball diameter, impact velocity and ball density based on simulation results of numerous impact cases (Fig. 3a-d). The following numerical relations are found: (a) the indent diameter $D$ has a linear relationship with ball diameter $d_{B}$, and a power-law relationship with both the normal impact velocity $v_{n}$ and ball density $\rho_{\mathrm{B}}$, as described in Eq. (3); (b) the indent depth $h$, which describes the roughness of the component surface, has a linear relationship with the ball diameter $d_{B}$ and normal impact velocity $v_{\mathrm{n}}$ and square-root law with the ball density $\rho_{\mathrm{B}}$, as described in Eq. (4):

$$
\begin{aligned}
& D \propto d_{B}, D \propto v_{n}^{1 / 2}, D \propto \rho_{B}^{1 / 4}, \\
& h \propto d_{B}, h \propto v_{n}, h \propto \rho_{B}^{1 / 2} .
\end{aligned}
$$

The indent depth dependencies in Eq. (4) are consistent with the peak-to-valley value predicted for aluminum treated with a surface nano-crystalline and hardening process from the analytical model proposed by Dai et al. ${ }^{31}$ Additionally, the indent size can be found to have a relationship with the impact kinetic energy $E_{k}$ of the impact ball:

$\left\{\begin{array}{l}D \propto E_{k}^{1 / 4} \\ h \propto E_{k}^{1 / 2},\end{array}\right.$ for constant $d_{B}$,

where:

$$
E_{k}=\frac{\pi}{12} \rho_{B} d_{B}^{3} v_{\mathrm{n}}^{2} \text {. }
$$

The value of the indent diameter and the indent depth can be further numerically expressed as:

$$
\begin{aligned}
& D=k_{1} \cdot\left(d_{B}\right) \cdot\left(v_{n}\right)^{1 / 2} \cdot\left(\rho_{B}\right)^{1 / 4}, \\
& h=k_{2} \cdot\left(d_{B}\right) \cdot\left(v_{\mathrm{n}}\right) \cdot\left(\rho_{B}\right)^{1 / 2}, \\
& \text { or } \\
& D=k_{3} \cdot\left(d_{B} \cdot E_{\mathrm{k}}\right)^{1 / 4}, \\
& h=k_{4} \cdot\left(E_{k} / d_{B}\right)^{1 / 2},
\end{aligned}
$$

where $d_{\mathrm{B}}, v_{n}, \rho_{\mathrm{B}}$ and $E_{\mathrm{k}}$ are the ball diameter, normal impact velocity, ball density and impact kinetic energy, respectively, with units listed in Table 3. $k_{1}, k_{2}, k_{3}$ and $k_{4}$ are material constants. Based on our study, we find that $k_{1}, k_{2}, k_{3}$ and $k_{4}$ remain almost constant; their average values for AISI $316 \mathrm{~L}$ stainless steel, obtained from the simulation results, were $k_{1}=k_{1 \text { average }}=40$, $k_{2}=k_{\text {2average }}=0.35, k_{3}=56$, and $k_{4}=0.68$. Figure $3 a-d$ illustrates the simulated points and fitting curves that are fit well by Eqs. $(7,8)$.

The newly developed model can also be applied to other structural materials, such as AISI $304 \mathrm{~L}$ stainless steels, where the indent size generated by single normal impact again satisfies the proposed function in Eqs. $(7,8)$, with the constants found to be $k_{1}$ $=36.8, k_{2}=0.29, k_{3}=51.5$, and $k_{4}=0.57$. The model was further verified by the equivalence of the numerical solutions with experiments for the indent depth, specifically the peak-to-valley values, for surface nano-crystallized and hardened 5052 aluminum plates $^{31}$ where a value of $k_{2} \approx 0.71$ was found. ${ }^{32}$

Numerical solutions for indent size under random impact

The normal impact model described above can be readily extended to impacts at oblique angles, where the oblique impact angle $\theta$ is defined between the impact direction and the horizontal plane in 3-D space, as shown in Fig. 3e. First, the material parameters $k_{1}$ and $k_{2}$ in Eqs. $(7,8)$ must be verified for impacts at oblique angles. Here, the value of the indent diameter and the indent depth can be expressed as:

$$
\begin{aligned}
& D=k_{1} \cdot\left(d_{B}\right) \cdot(v \cdot \sin \theta)^{1 / 2} \cdot\left(\rho_{B}\right)^{1 / 4}, \\
& h=k_{2} \cdot\left(d_{B}\right) \cdot(v \cdot \sin \theta) \cdot\left(\rho_{B}\right)^{1 / 2} .
\end{aligned}
$$

With the same ball size, $d_{\mathrm{B}}=3 \mathrm{~mm}$, same ball density, $\rho_{\mathrm{B}}=8.0 \mathrm{~g} /$ $\mathrm{cm}^{3}$ (equivalent to $8,000 \mathrm{~kg} / \mathrm{m}^{3}$ ), and same magnitude of impact velocity $v=10 \mathrm{~m} / \mathrm{s}$, the profiles of indentations induced by impacts at different oblique angles are shown in Fig. 3f, where $h$ and $h^{\prime}$ are two values of the indent depth due to the non- 

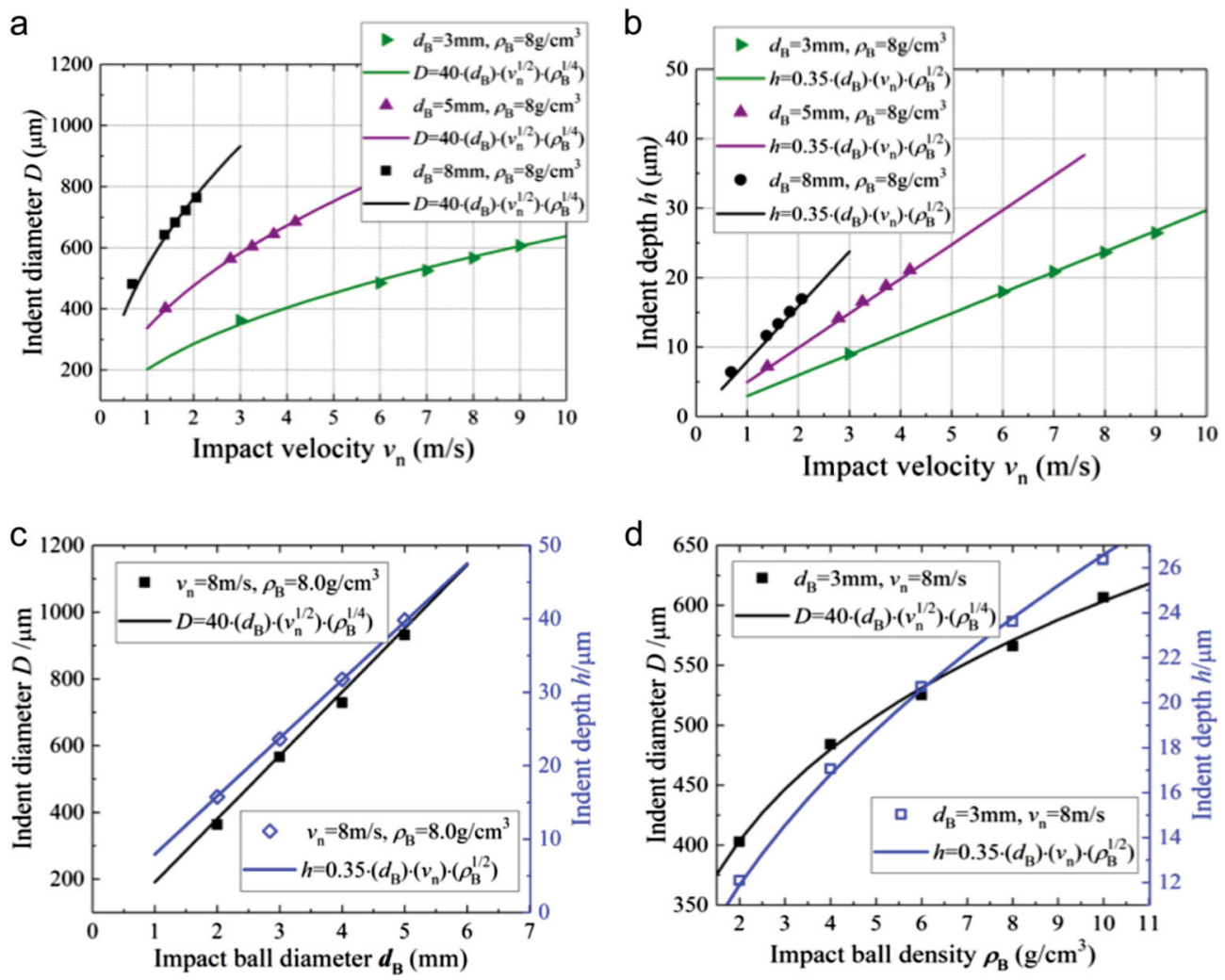

e

f
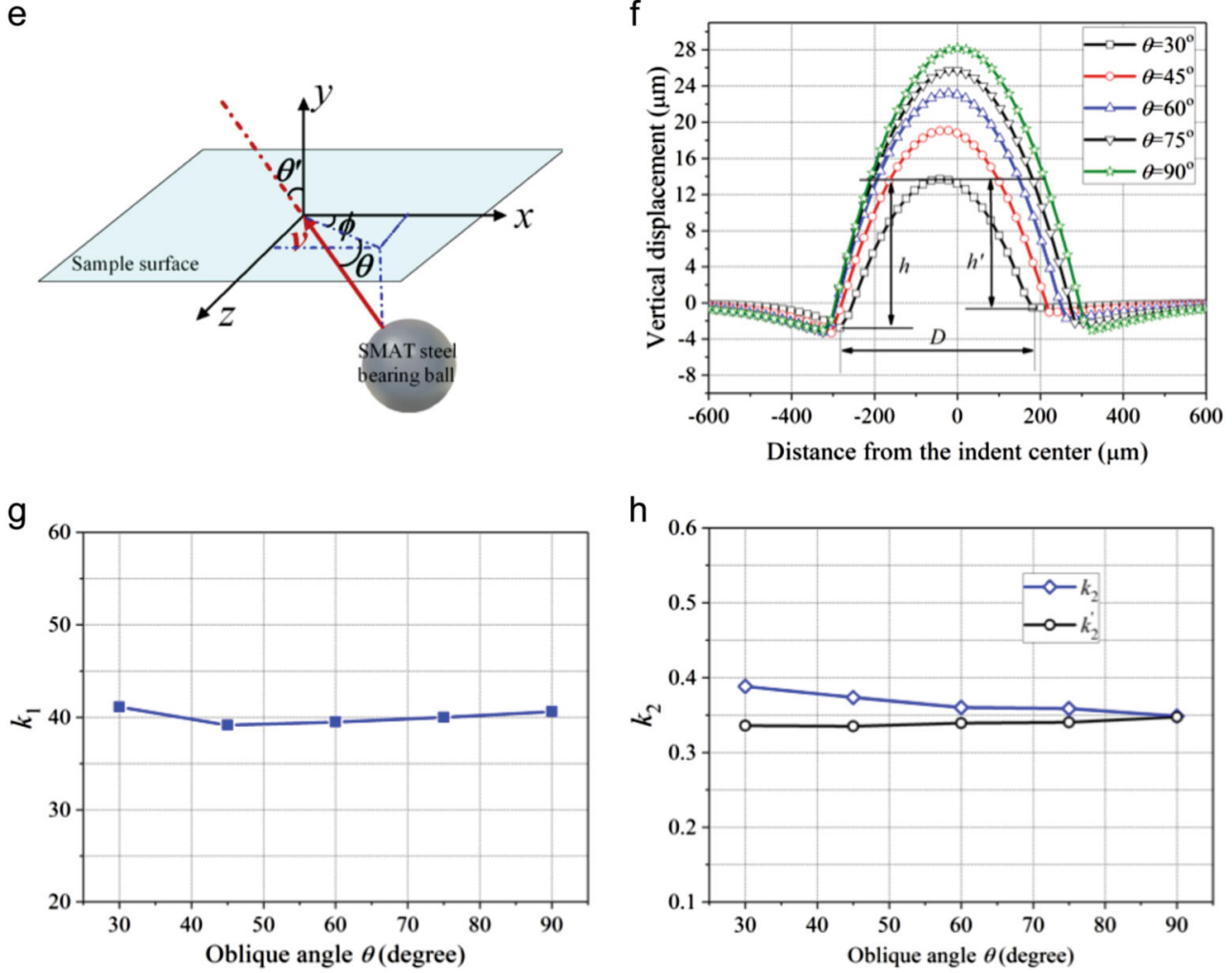

Fig. 3 Analysis of the effects of ball parameters on the indent size: a-d illustrates the simulated points and fitting curves for the indent diameter and indent depth, generated by a single normal impact, as a function of the ball parameters, respectively; $\mathbf{a}$, $\mathbf{b}$ show the indent diameter and indent depth, as a function of the ball impact velocity $v_{n}$, respectively; $\mathbf{c}$ shows the relationship between the indent size (diameter and depth) and impact ball diameter $d_{B}$; $\mathbf{d}$ shows the relationship between the indent size (diameter and depth) and impact ball density $\rho_{\mathrm{B}} ; \mathbf{e}$ is a 3-D illustration of the impact model with an oblique impact angle $\theta$. The angle $\theta$ is defined as the angle between the impact direction and the horizontal plane in 3-D space. $\mathbf{f}$ Profiles of indentations induced by impacts with different oblique angles, where $h$ and $h^{\prime}$ are two values of indent depth due to the non-symmetry of indent shape induced by oblique impact. $\mathbf{g}, \mathbf{h}$ Calculation of the constants $k_{1}, k_{2}$ and $k_{2}{ }^{\prime}$ using proposed numerical solutions (Eqs. $(11,12)$ ); these constants are found to be effectively constant with impact angle $\theta$ 
Table 3. Units of the parameters in Eqs. (7-10)

\begin{tabular}{lllllll}
\hline Parameters & $D$ & $h$ & $d_{\mathrm{B}}$ & $v_{n}$ & $\rho_{\mathrm{B}}$ & $E_{k}$ \\
\hline Unit & $\mu \mathrm{m}$ & $\mu \mathrm{m}$ & $\mathrm{mm}$ & $\mathrm{m} / \mathrm{s}$ & $\mathrm{g} / \mathrm{cm}^{3}$ & $\mathrm{~J}$ \\
\hline
\end{tabular}

symmetrical shape of indents resulting from oblique impacts. As seen in Fig. $3 \mathrm{~g}$, h, the values of the corresponding constants $k_{1}, k_{2}$ and $k_{2}{ }^{\prime}$ for impacts at different oblique angles are almost the same with those values for the normal impact case. Different indent sizes with different oblique impact angles can now be predicted both for SMAT and, by comparison, for shot peening. As the impact angle in shot peening tends to be uniform, Eq. (3) can be used directly; for SMAT, conversely, the impact angle $\theta$ is random, $\theta \in\left[0^{\circ}, 90^{\circ}\right]$, such the average indent size $\bar{D}_{\text {SMAT }}$ and $\bar{h}_{\text {SMAT }}$ can be evaluated as:

$\bar{D}_{\mathrm{SMAT}} \approx \gamma_{D} \cdot k_{1} \cdot\left(d_{B}\right) \cdot(v)^{1 / 2} \cdot\left(\rho_{B}\right)^{1 / 4}$,

$\bar{h}_{\mathrm{SMAT}} \approx \gamma_{\boldsymbol{h}} \cdot k_{2} \cdot\left(d_{B}\right) \cdot(v) \cdot\left(\rho_{B}\right)^{1 / 2}$,

where $\gamma_{D}=\frac{\int_{0}^{90}(\sin \theta)^{1 / 2} \mathrm{~d} \theta}{90} \approx 0.76$, and $\gamma_{h}=\frac{\int_{0}^{90}(\sin \theta) \mathrm{d} \theta}{90} \approx 0.64$. Eq. (14) can then be used to give a quantitative prediction of surface roughness of a material following SMAT treatments.

Experimental measurements

The ball size and density, used in Eqs. $(7,8)$, are easily measured and controlled, whereas the impact velocity cannot be controlled directly in SMAT experiments because the motion of balls is propelled by the vibration of the ultrasonic horn surface in random fashion (Fig. 4a). To validate these solutions (Eqs. $(7,8)$ ), the following experiment was designed to determine whether such numerical simulations using finite element methods are capable of predicting accurate indent sizes.

The velocities of the flying balls were measured by taking consecutive frame photographs with a high-speed camera (Phantom VR706) at the rate of 5,000 frames per second. The delay time of each frame was $0.2 \mathrm{~ms}$ so that the impact velocity could be calculated by counting the number of frames a ball travels from the bottom of the chamber to the sample surface for a fixed distance of $25 \mathrm{~mm}$. Impingements were recorded by the high-speed camera. As an example, 16 frames were captured during the flight of a 3-mm steel ball onto the sample surface; four typical images are shown in Fig. $4 \mathrm{~b}$. The resulting vertical velocity distribution of the selected balls for multiple impacts, shown in Fig. $4 \mathrm{c}$, gives an average velocity of $7.0 \mathrm{~m} / \mathrm{s}$.

The measured diameters of the indents on the surface of the SMAT-treated AISI $316 \mathrm{~L}$ stainless steel are shown in Fig. $4 d$. Choosing the larger circular dimples because they were more likely induced by normal impacts, the average diameter from measurements on 100 indents was $515 \mu \mathrm{m}$, consistent with an average indent diameter of $525 \mu \mathrm{m}$ predicted by the FEM simulation and $531 \mu \mathrm{m}$ from the analytical model of Eq. (7). There is approximately a $3 \%$ difference between the experimental value and that calculated by the proposed numerical solutions.

\section{Plastic zone under oblique impacts}

Considering the oblique impact model with the same ball size $d_{\mathrm{B}}$ $=3 \mathrm{~mm}$, ball density $\rho_{\mathrm{B}}=8,000 \mathrm{~kg} / \mathrm{m}^{3}$, and initial normal velocity $v_{\mathrm{n}}=10 \mathrm{~m} / \mathrm{s}$, the contours of the displacement along the thickness (y-direction) for different oblique angles are shown in Fig. 5b-f. The area under each impact is extruded to form peaks (designated in blue) and valleys (in red). The percentage of the pile-up area at the rim of the indent increases with increasing oblique angle; by contract, normal impacts lead to a symmetrical shape of indent.
Figure $5 \mathrm{~g}$ shows the distribution of equivalent plastic (Mises) strain, $\bar{\varepsilon}$, along the depth of the indent for different oblique impact angles, where $\bar{\varepsilon}=\frac{1}{3 \sqrt{2}}\left(\left(\varepsilon_{x x}-\varepsilon_{y y}\right)^{2}+\left(\varepsilon_{y y}-\varepsilon_{z z}\right)^{2}+\left(\varepsilon_{z z}-\varepsilon_{x x}\right)^{2}\right.$ $\left.+6\left(\varepsilon_{x y}^{2}+\varepsilon_{y z}^{2}+\varepsilon_{z x}^{2}\right)\right)^{1 / 2}$. These numerical results in Fig. 5 show that the location of the maximum equivalent plastic strain is not at the actual surface of the impact, but slightly below it. They also clearly reveal that impacts generated with the same normal impact velocity, $v_{n}$, will induce the same degree of plastic deformation beneath the impact point. The plastic strains are non-symmetric for impacts at an oblique angle. The equivalent plastic strain, shown in Fig. 5, represents the equivalent plastic strain distribution along the depth beneath the impact point. Specifically, the depth of the material influenced by plastic deformation under such impacts, $r_{y}$, can be computed to be approximately equal to the indent diameter $D$, if the onset of plastic deformation is defined to occur when the equivalent plastic strain reaches a certain critical value of $\bar{\varepsilon}=0.2 \%$. Accordingly:

$r_{y} \approx k_{1} \cdot\left(d_{B}\right) \cdot(v \cdot \sin \theta)^{1 / 2} \cdot\left(\rho_{B}\right)^{1 / 4}$.

For impacts at random oblique angle $\theta$, as in SMAT, the average depth of plastic zone can be calculated as $\bar{r}_{y}^{\text {SMAT }} \approx \gamma_{l} \cdot k_{1} \cdot\left(d_{B}\right)$. $(v)^{1 / 2} \cdot\left(\rho_{B}\right)^{1 / 4}$ with $\gamma_{l} \approx 0.76$. This result has significant importance in estimating the plastic zone influenced by impact deformation.

Analytical evaluation of SMAT coverage

In order to control the process of the surface modification treatment accurately so that sufficient indent coverage can be attained, it is important to know the relationship between impact ball parameters and the indent coverage on a sample surface. This section focuses on how to develop such relationships using the three-dimensional impact model depicted in Fig. 3e. To do this, the velocity components are first redefined in Eq. (16) as:

$\left\{\begin{array}{l}v_{x}=v \cdot \cos \theta \cdot \cos \phi \\ v_{y}=v \cdot \sin \theta \\ v_{z}=v \cdot \cos \theta \cdot \sin \phi\end{array} \quad\left(0^{\circ}<\theta \leq 90^{\circ}\right)\right.$.

\section{Global coverage rate of impacts}

The impact process associated with SMAT is random, which implies that some regions of the sample surface may receive more rigid ball impacts while others less. However, by increasing the duration of the treatment, it can be presumed that the entire component surface will eventually be covered by indents. To analyze this, a random impact model was developed to investigate the statistical characteristics of impacts using MATLAB. We used a sample to be SMAT-treated with a surface diameter of $70 \mathrm{~mm}$, and made the following assumptions in the model: (a) the impact events are independent from each other, which means (i) any interaction of flying balls before they impact the surface is not considered, and (ii) an impact event is modeled to take place only after the preceding impact event is complete, i.e., from plastic deformation to elastic recovery; (b) the impact velocity for each flying ball is identical, (c) the oblique impact angles are random; (d) the indent size created by each impact is different but can be calculated by the proposed numerical solution in Eq. (11).

The simulation results are presented in Fig. 6. After 1000 impacts by $8-\mathrm{mm}$ balls, some parts of the surface still have not been covered by indents (Fig. 6a-c), while after 20,000 impacts, the entire plate surface is covered (Fig. $6 \mathrm{~d}-\mathrm{f}$ ). To investigate, as a function of ball parameters, the percentage of indent coverage, specifically the ratio of indented area to total sample surface area, impact processes with identical (Fig. $6 \mathrm{~g}$ ) and random oblique angles (Fig. $6 \mathrm{~h}$ ) were respectively modeled. The scatter points in 
a

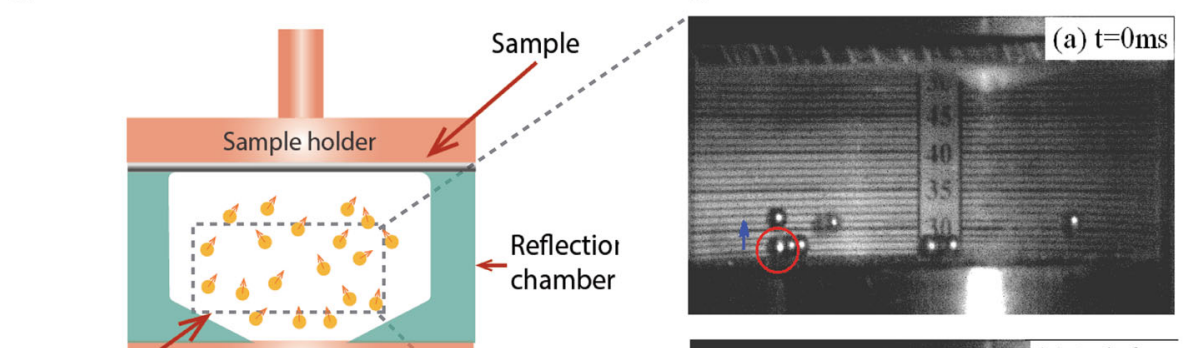

b

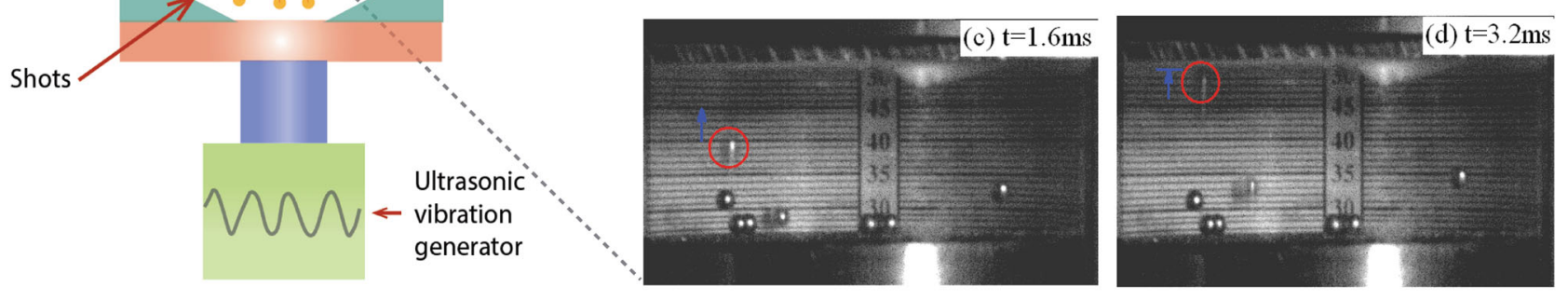

C

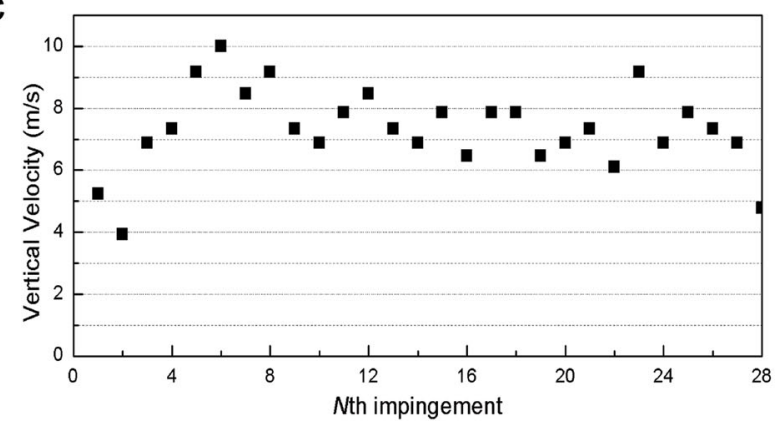

d

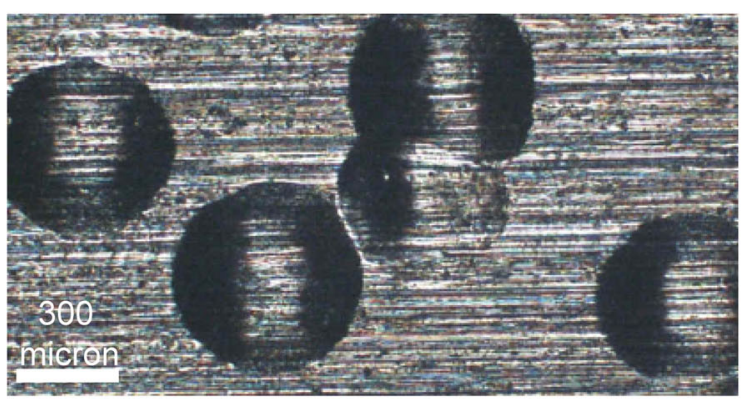

Fig. 4 Experimental methods used to calibrate the proposed numerical solutions of indent size: a Ultrasonic-assisted surface mechanical attrition treatment setup. b Four photographs representative of multi-frame images of 3-mm steel balls during SMAT treatment on AISI $316 \mathrm{~L}$ stainless steel during one impact duration taken by a high-speed camera with rate of 5,000 frames per second. c Distribution of the vertical velocity of the 3-mm steel balls during the SMAT treatment of the stainless-steel plate. d Image of the impacts and their typical diameters measured with an optical metallurgical microscope

Fig. $6 \mathrm{~g}$, $\mathrm{h}$ show the percentage of area covered by indents, the coverage ratio, $C R$, vs. the total number of impacts $N$. Seven fitting curves in Fig. $6 \mathrm{~g}, \mathrm{~h}$ all satisfy the following type of Avrami equation:

$1-C R=\exp \left(-k_{C} N\right)$,

where $k_{C}$ is a fitting parameter related to the indent area $\left(S_{\text {indent }}\right)$ for a single impact and the sample surface area $\left(S_{\text {plate }}\right)$, given in terms of a constant $a$ by:

$k_{C}=a \frac{S_{\text {indent }}}{S_{\text {plate }}}$.

By substituting Eqs. (11, 18) into Eq. (17), the relationship between the indent coverage and impact ball parameters can be obtained as:

$C R=1-\exp \left\{-a \frac{\pi \cdot k_{1}^{2}\left(d_{B}\right)^{2} \cdot(v \cdot \sin \theta) \cdot\left(\rho_{B}\right)^{1 / 2}}{4 S_{\text {plate }}} N\right\}$.

The simulation results in Fig. $6 \mathrm{~g}$ give a value of $a$ of unity provided the impact angle $\theta$ is uniform, e.g., as in shot peening. Figure $6 \mathrm{~h}$ shows the corresponding predictions for the coverage rate as a function of the number of random oblique angle impacts, i.e., for SMAT. The coverage ratio for SMAT can be expressed as:

$C R_{\text {SMAT }}=1-\exp \left\{-\gamma_{C} \frac{\pi \cdot k_{1}^{2}\left(d_{B}\right)^{2} \cdot(v) \cdot\left(\rho_{B}\right)^{1 / 2}}{4 S_{\text {plate }}} N\right\}$,

where $\gamma_{c}$ was obtained from the fitting curves to be equal to 0.76 , and $\gamma_{C}=\gamma_{D}$ because the coverage rate is directly related to the average indent size.

Local evaluation of the SMAT impact frequency

To construct a method for calculating the effective coverage, we envisage a model where randomly distributed impacts are assumed to occur on a two-dimensional circular area $S_{\text {plate }}$ with radius $R_{\text {plate }}$ (based on the size of our SMAT fixture), as shown in Fig. 6i. Each circle here represents an indent. The diameter $D$ of the indent can be determined by Eq. (11) for a given set of ball parameters. We take the center points of the indents $\left(P=\left[P_{1}, P_{2}\right.\right.$, $\left.\left.P_{3}, \ldots, P_{i}, \ldots, P_{N}\right]\right)$ to have coordinates $\left(x_{i}, y_{i}\right), i=1,2,3, \ldots, N$.

Assuming that a location $P_{\mathrm{S}}$ with a coordinate $\left(x_{\mathrm{s}}, y_{\mathrm{s}}\right)$ on the sample surface will be impacted by the ith impingement at a random oblique angle if the distance $d_{s i}$ between $P_{\mathrm{s}}$ and the center point $P_{\mathrm{i}}$ of the indent is less than or equal to $D_{\mathrm{i}} / 2$, where $D_{\mathrm{i}}$ is the diameter of the indent generated by the $i^{\text {th }}$ impingement and calculated by Eq. (11), it follows that:

$d_{S i}=\sqrt{\left(x_{S}-x_{i}\right)^{2}+\left(y_{s}-y_{i}\right)^{2}} \leq D_{i} / 2$

Accordingly, the $N$-sequenced field variable $\eta_{P_{S}}=$ $\left(\eta_{P_{s}}^{1}, \eta_{P_{s}}^{2}, \eta_{P_{s}}^{3}, \ldots, \eta_{P_{s}}^{i}, \ldots, \eta_{P_{s}}^{N}\right)$ can be generated depending on whether the position $P_{S}$ is impacted by the flying balls or not. Similar to the diffuse-interface model (Fig. 6j) in classical phasefield theory, ${ }^{33}$ the field variables $\eta_{P_{S}}$ should be continuous 

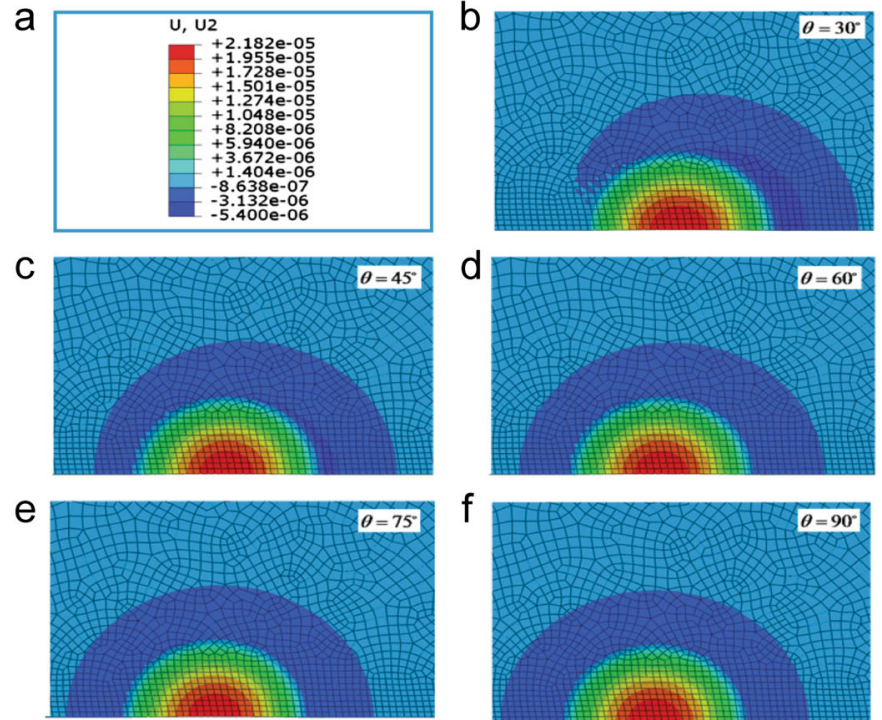

9

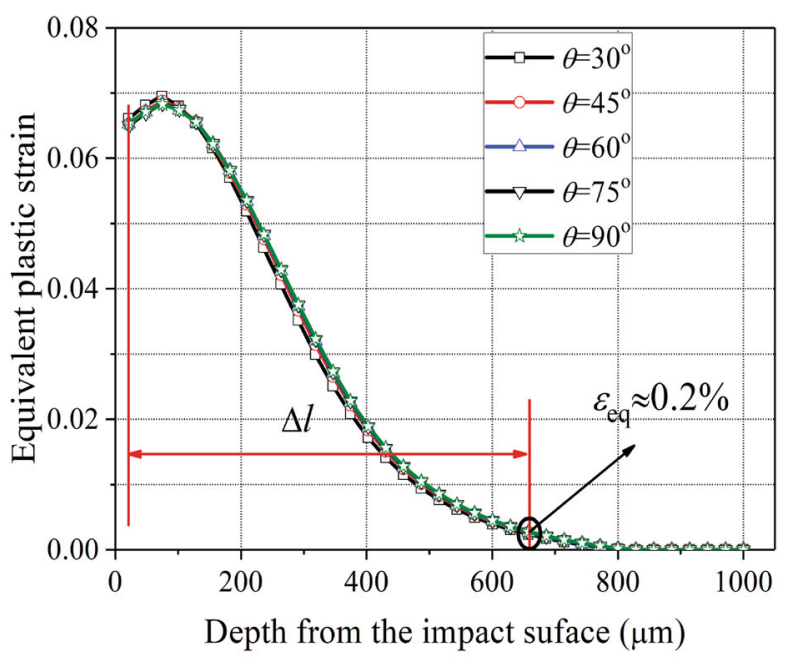

Fig. 5 Study of the relationship between the plastic zone and the random impact ball parameters: a-f Contours of the displacement $U$ along thickness direction (U2) with different impact angles $\theta=30^{\circ}, 45^{\circ}, 60^{\circ}, 75^{\circ}$ and $90^{\circ}$, respectively (unit of $U$ is meters). The area under impact is extruded to form peaks (blue) and valleys (red). The percentage of the pile-up area increases with the increase in the oblique impact angle. The symmetrical indent shape is generated only by the normal impact, and the maximum U2 of $22 \mu \mathrm{m}$ is the same as the value of the indent depth. g Equivalent plastic strain, $\bar{\varepsilon}$, distribution along depth at different oblique angles but with the same normal velocity $v_{n}$. The depth influenced by plastic deformation under such impact, $\Delta l$, is approximately equal to the indent diameter $D$ if the plastic deformation of a material is defined to begin when the equivalent plastic strain reaches a certain critical value of $\bar{\varepsilon}=0.2 \%$

functions between 0 and 1 depending on the percentage of the area covered.

Since each indent size is rather small compared with the whole sample surface, by a factor of between $\sim 10^{-5}$ and $10^{-4}$, the field variable $\eta_{P_{S}}$ can be assumed to be a step function, i.e., with a discontinuous sharp interface, as shown in Fig. 6k for only two values, 1 or 0 , for simplification:

$\eta_{P_{S}}^{i}= \begin{cases}1, & d_{S i} \leq D_{i} / 2 \\ 0, & \text { elsewhere }\end{cases}$

The number of impacts $n_{\mathrm{s}}$ received by a randomly selected position $P_{\mathrm{S}}$ can then be defined as:

$n_{\mathrm{S}}=\sum_{i=1}^{N} \eta_{P_{\mathrm{S}}}^{i}$

We further find that the probability $(P(x))$ of one surface point receiving $x$ number of impacts can be characterized by a normal distribution, as shown in Fig. $6 \mathrm{l}, \mathrm{m}$ and expressed as:

$P(x)=a \cdot \exp \left(-\frac{(x-\bar{n})^{2}}{2 c^{2}}\right)$,

where $a$ and $c$ are real constants and $\bar{n}$ is the average number of impacts. For a $3-\mathrm{mm}$ ball $\left(v=8.0 \mathrm{~m} / \mathrm{s}, \rho_{\mathrm{B}}=8,000 \mathrm{~kg} / \mathrm{m}^{3}\right)$ impacting an AISI $316 \mathrm{~L}$ stainless steel $70-\mathrm{mm}$ diameter plate, the indent diameter can be calculated by Eq. (11), with the number of impacts obtained from Eqs. $(19,20)$.

The statistical characteristics of the impact frequency for different types of impact, i.e., at uniform impact and random oblique impact angles, are shown respectively in Fig. $6 \mathrm{l}, \mathrm{m}$. If full coverage is defined to be equivalent to $99 \%$ of the actual coverage, it is clear that points on the plate must be impacted on the order of 4 to 5 times to achieve full coverage under normal impacts, whereas for impacts at random oblique angles, which is the situation during SMAT treatments, each point would need around 11 to 12 hitting impacts.
Full-coverage multiple-impact model

Based on the analysis described above, clearly the process of SMAT demands multiple impacts to attain full coverage. Accordingly, here we develop a full-coverage multiple-impact model to describe this scenario. Figure $7 a, b$ presents the modeling considerations for multiple impingements which consider simultaneously full coverage, the location of random impacts and oblique angle impacts. Each circle in Fig. 7a represents an indent induced by one impact, with the circle diameters calculated using Eq. (11). For the random impacts during SMAT, the global full coverage is generated from Eq. (19), as illustrated in Fig. 7a. It is apparent that the indent sizes are different from each other due to the differing oblique angles of impact. For computational efficiency, only the local full-coverage model is transferred into the finite element formulation (Fig. 7b), where we consider a total of 64 balls, all with a diameter of $3 \mathrm{~mm}$. Using this local fullcoverage, random multiple-impact model, the impact location and impact angle of each ball are obtained from the corresponding global coverage using Eqs. (17-24). For such random, multiple impacts, we consider the overlap of indents by explicitly analyzing the first and second impacts. Specifically, the second ball is considered to impact the concave region of the first indent. Multiple impacts involving complex mechanical and multi-axial loading conditions result in strain hardening and high strain-rate hardening under the plastic deformation. To explain how we handle this, we have developed the flow chart to illustrate how our model for full coverage multiple impacts works and how it is coupled to the finite element analysis. This is described in the Methods section with the flow chart shown in Fig. 8.

The contours of the resulting surface roughness were derived (Fig. 7c) and show clear indents with a spherical base generated by the impacts of the balls. The sizes of the indents are different, however, and exhibit some sharp ridges on the side of impacting surface.

We also investigated the states of accumulated plastic strain using the random multiple-impact model. Specifically, by consideration of the concave geometry caused by the previous impact, the equivalent plastic strain distribution on the impact 
surface induced by four successive random impacts during the SMAT process was calculated; results are shown in Fig. $7 \mathrm{~d}$. It is apparent from this figure that the sharp ridges of the indent induced by the first impact are changed by the second impact. The random multiple impact model described in this paper attempts to simulate the real random impact process in three- dimensional space; it therefore provides predictions of the strain as well as the geometry of the indents on the impact plane. The oblique angles of impact and the impact locations are both modeled as random; however, the impact angle and location of each impact are recorded for one full-coverage random impact process and such data are then used to build-up the local finite
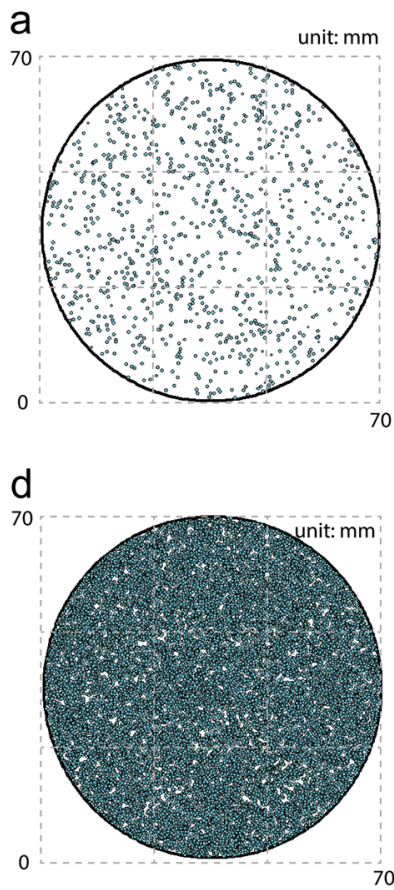

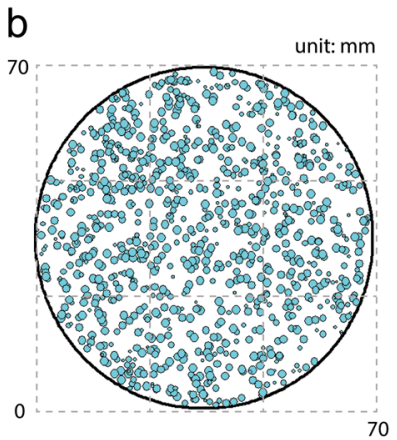

e

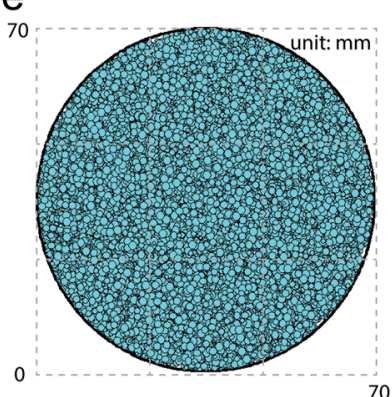

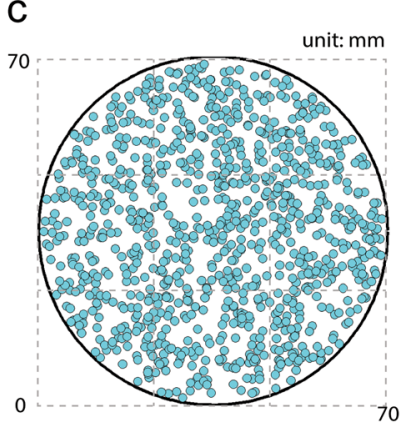

f

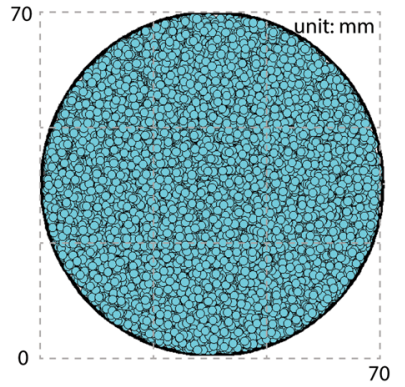

9

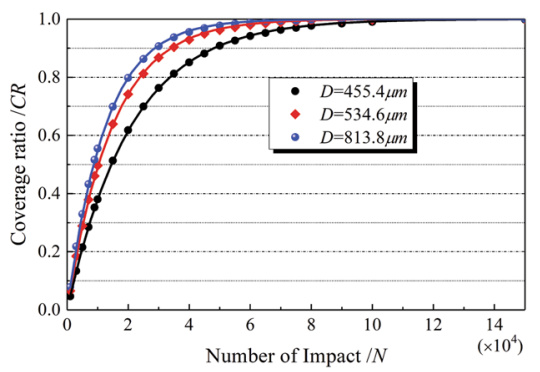

$\mathrm{h}$

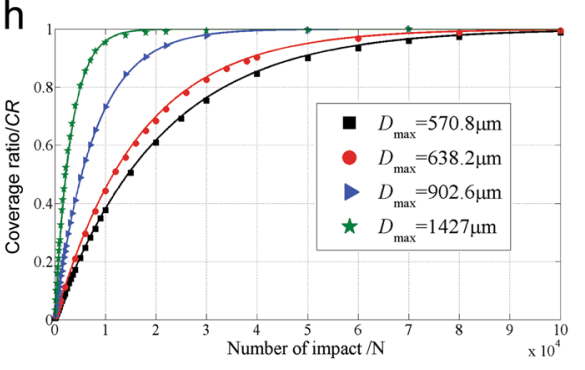

K

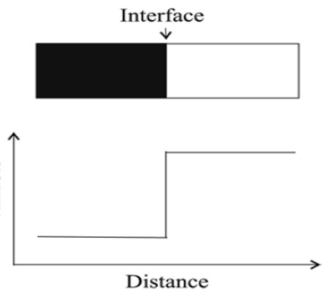

I

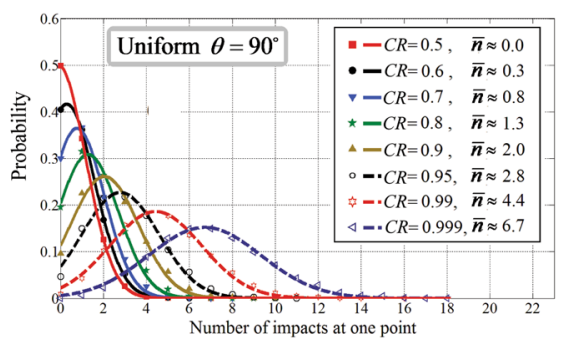

j

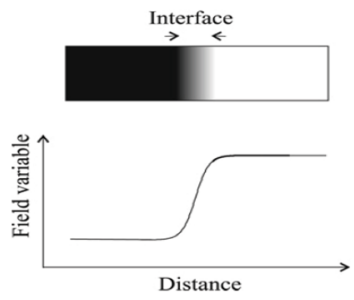

$\mathrm{m}$

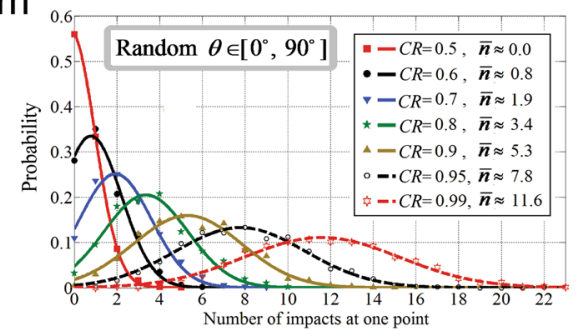


Fig. 6 Analytical evaluation of coverage for SMAT process: Simulation results from the random impact model with 1,000 indents induced by a 3-mm, b. 8-mm rigid balls with random impact angles, and c 8-mm rigid ball at an angle $\theta=90^{\circ}$, and for 20,000 indents induced by d. 3-mm, e $8-\mathrm{mm}$ rigid balls with random impact angles, and $\mathbf{f} 8-\mathrm{mm}$ rigid ball at an angle $\theta=90^{\circ}$. For both the 1000 and 20,000 impact cases, each small circle represents an indent produced by one impact. For indents generated by balls with same impact angle, as in shot peening, the indent size is uniform and the relationship between the coverage rate and the number of impacts is shown in $\mathbf{g}$. For indents generated by balls with random impact angles, the indent size created by each impact is different with each other and calculated by the proposed numerical solution (Eq. (11)). The maximum indent size is induced by normal impacts. $\mathbf{h}$ Coverage rate as a function of impact numbers for SMAT (random impact angle). i Schematic illustration of indents created by random impact. For evaluation of the local impact frequency, the classical phase field model $^{33}$ is employed to calculate whether the position is impacted by the flying balls. $\mathbf{j}$ Diffuse-interface model and $\mathbf{k}$ sharp-interface model, which illustrate the values of the field variables $\eta_{P_{S}}$ to be 1 or 0 depending on whether the position $P_{S}$ is impacted by the flying balls. Statistical characteristics of impact frequency under random impacts for $\mathbf{I}$. uniform angles and $\mathbf{m}$. random angles

element model to investigate and calculate the material behavior under such random impacts. To illustrate the geometry of the deformation process during multiple impacts, the contours of displacement on the impact surface are shown in Fig. 7c, where the final geometry is represented by the contours of the resulting surface roughness. The contours of equivalent plastic strains in the cross section of plate during the impact process are illustrated in Fig. 7e, f. Whereas the maximum equivalent plastic strain is calculated to be $\sim 0.1$ beneath one single normal impact (Fig. 7e), after random multiple impacts, the maximum equivalent plastic strain is increased by more than a factor of three to 0.36 (Fig. 7f). The distribution of such equivalent plastic strain in the depth direction beneath the center of the local impact zone is plotted in Fig. $7 \mathrm{~g}$. It is interesting to note here that if the onset of plastic deformation is defined to occur when the equivalent plastic strain reaches a certain critical value of $\bar{\varepsilon}=0.2 \%$, the depth of the deformation surface layer under a single impact at one location and that after random multiple impacts stays essentially unchanged. In fact, for such random multiple impacts, each point would receive some 11 to 12 impacts, as shown in Fig. $6 \mathrm{~m}$. The fundamental reason why the equivalent strains caused by a single impact vs. multiple impacts are almost identical below a depth of $280 \mu \mathrm{m}$ is that the material displays strong strain hardening. As the incident energy of the ball is limited, the maximum strain levels saturate after only a small number of impacts. Our analysis also predicts that the depth of the material influenced by plastic deformation is approximately equal to the indent diameter $D$, as given in Eq. (15). Thus, from a practical perspective, if a larger depth of the deformed layer is required, one approach would be to utilize higher-density balls with a larger diameter to impact the target surface at high velocity. For example, in some treatment cases, 8-mm diameter balls with a larger density have been employed precisely for this purpose. However, the magnitude of the plastic strains and the width and total volume of the zone of plastic deformation induced by random multiple impacts will be naturally larger than that induced by a single impact at one point.

The effect of random multiple collisions on the equivalent flow stress is shown in Fig. 7h. The flow stress calculated on the assumption of full coverage and multiple impacts is clearly much larger than the corresponding stress calculated assuming successive multiple impacts at one location. We have also determined the influence of the number of impacts as well as the random impact angles on the value of the equivalent flow stress; the flow stress shown in Fig. $7 \mathrm{~h}$ is the steady-state value. Our results indicate that adjacent random impacts markedly influence the resulting stress distribution. Thus, a multiple-impact model has been developed that can be utilized to simulate the evolution of mechanical properties as a function of the processing parameters associated with SMAT. Currently, in our analysis, the full-coverage random multiple-impact model for the SMAT process is statistical in nature. The random impact location and random impact angle for each impact are recorded until the impact surface is fully covered by indents. We believe that these models can be used as an efficient simulation tool to provide a high-fidelity understanding of the driving force for nano-crystallization. Moreover, the approach can be readily extended to any metallic material, even with complex shapes.

\section{DISCUSSION}

The surface modification technique of surface mechanical attrition, or SMAT, involves the high-speed impact of small hard balls onto a metal surface to induce surface nano-crystallization and consequent graded microstructures and hardening in structural steels and other metals. To aid the successful use of this technique, in this work analytical and numerical solutions for the indent size, indent depth, extent of local plasticity and impact coverage, as a function of the process parameters of ball size, impact velocity, ball density and kinetic energy, have been proposed and developed. Indeed, we derive both single impact and full-coverage random multiple-impact models for these scenarios. Our analyses reveal that during such impact-induced surface nano-crystallization, the indent diameter generated by a single impact displays a linear relationship with ball diameter and a power-law relationship with the normal component of impact velocity and ball density; conversely, the indent depth, which describes the roughness of the component surface, displays a linear relationship with ball diameter and impact velocity and a square-root relationship with ball density. We also derive the relationship between the extent of plasticity surrounding the indents and the parameters of ball impact process for random oblique impact angles that are characteristic of surface nanocrystallization techniques.

In general, we report here a newly developed model which we believe represents a viable approach for characterizing the mechanical effect of SMAT for a wide range of metallic materials. As indicated in the numerical solution, the material constants can be obtained for other materials using these same analytical formulas. As such, our analytical formulations can be readily applied to other metallic materials by employing any impact parameters to obtain the material constants. We believe that our results, which we verify by experiment, can provide a necessary foundation for an improved understanding of the SMAT procedure for metals, as well as for the further development of microstructural evolution in metallic alloys for improved strength, ductility, hardness and toughness.

\section{METHODS}

The procedures describing the general framework of how we implement our proposed full coverage multiple impacts model is described below, in particular to address strain hardening and high strain rate hardening under the plastic deformation, which we solve using finite element methods. Since the elastic-plastic material behavior is non-linear, the solutions are obtained using an iterative procedure which is shown schematically in Fig. 8. Each iteration of our procedure is comprised of two steps: i) a global step which, using the equilibrium equations, the elasticity law and the boundary conditions, consists of calculating the displacement at the nodes of the structure and the total strain and stresses, $\varepsilon$ and $\sigma$, at the Gauss points, assuming the increment of plastic strain $\Delta \varepsilon^{p}$ between time increments of $t$ and $t+\Delta t$ are known at all points, and ii) a local step involving a consideration of the total strain $\varepsilon$ (specifically its increment $\Delta \varepsilon$ ) 
a

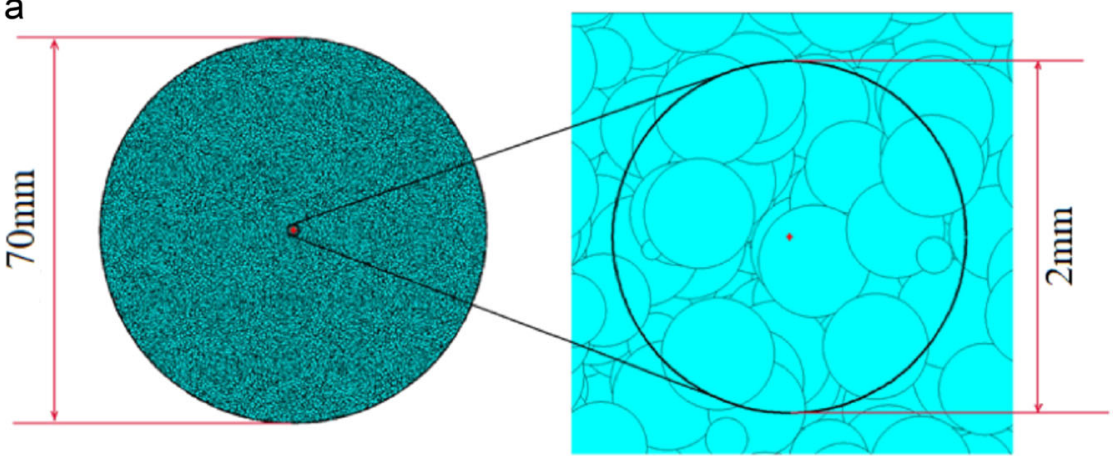

b

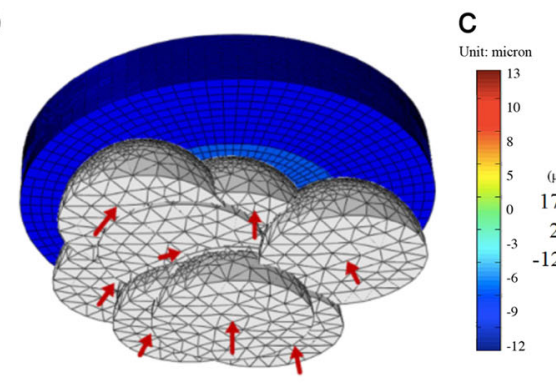

C

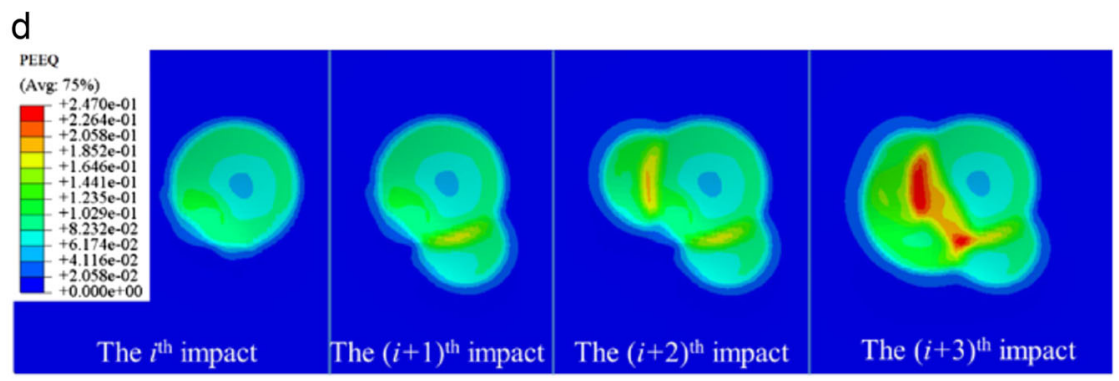

e

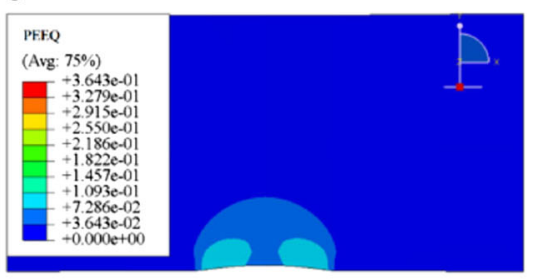

$f$
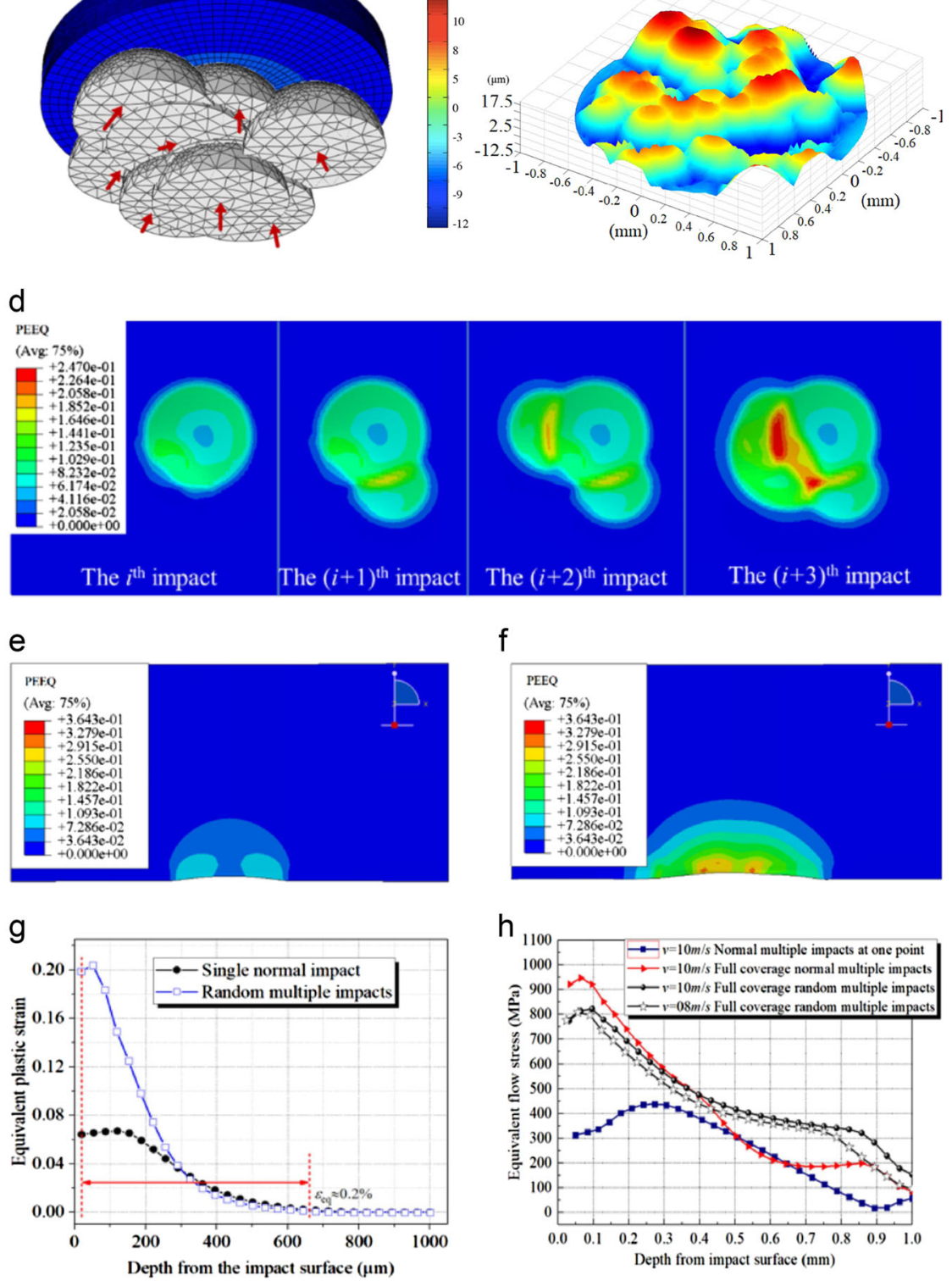

h

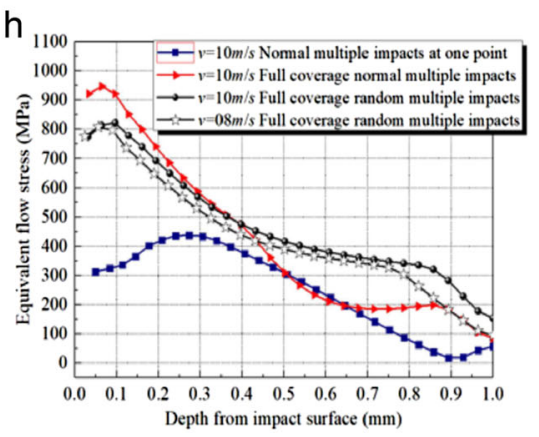

Fig. 7 Predicted results using a full-coverage, multiple random impact model: a The global full coverage model for random multiple impacts during SMAT based on Eqs. (11-24), each circle represents an indent induced by one impact; the indent sizes are different from each other due to the random oblique angles. $\mathbf{b}$ illustrates the local full-coverage finite element model. The impact point and impact angle of each ball are obtained from the global full-coverage model. c Contour plots of surface roughness using the local full-coverage finite element model where the vertical numbers indicate the depth of the indents in micrometers. $\mathbf{d}$ The equivalent plastic strain distribution on the impact surface, which induced by four successive random impacts. Contours of equivalent plastic strain distribution in the cross-section after e. a single normal impact, and $\mathbf{f}$ random multiple impacts. $\mathbf{g}$ Equivalent plastic strain, $\bar{\varepsilon}$, distribution along the depth from the impact surface; $\mathbf{h}$ corresponding equivalent flow stress distribution along depth after full coverage of random impacts 


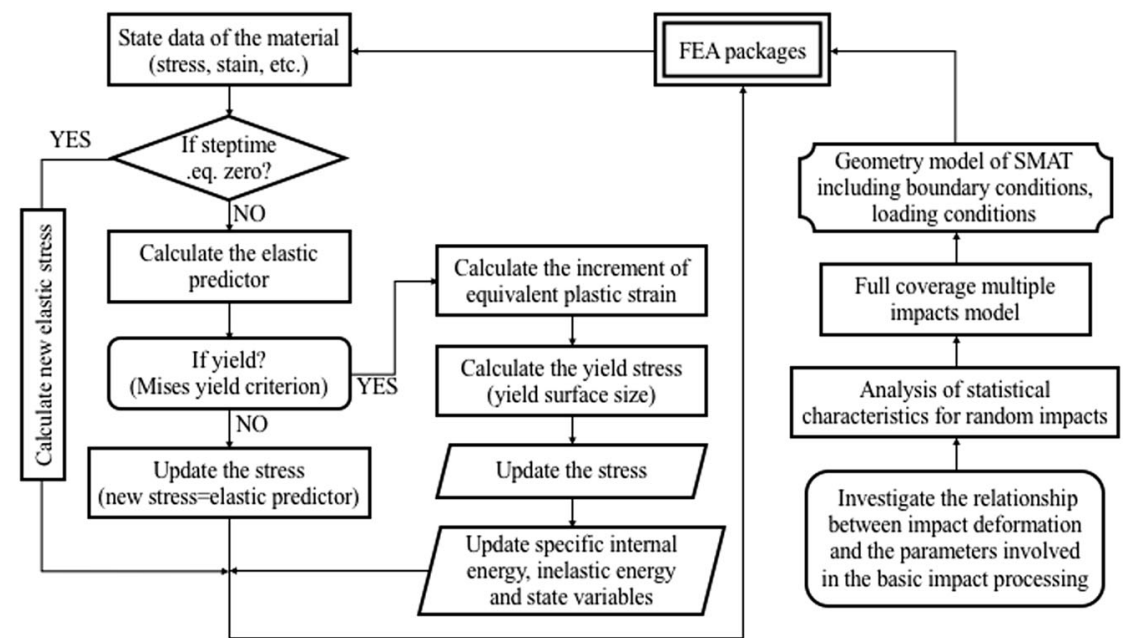

Fig. 8 Flow chart of our solution for the role of plasticity implicit in our proposed full coverage multiple impacts model

resulting from the global step at each point, i.e., calculation of the increments of elastic and plastic strains $\Delta \varepsilon^{e}, \Delta \varepsilon^{p}$ and the stresses $\sigma$ using the equations of plasticity. Programming the global step is a complex task, as it is independent of the applied plastic model and is totally performed by the commercial FEA package. However, the local step represents finite increments with the integration algorithm required to satisfy the material equations. The stress, elastic and plastic strains and hardening parameters must be calculated, stored and updated.

The first operation of the material behavior calculation is focused on the formulation of the elastic constant matrix, zero temporary variables, and setting the convergence tolerance. Starting from an equilibrium state at time $t$, the material equations are all satisfied and all quantities (stress, strain, etc.) at the integration point are known from the global FEA analysis. The total strain increment $d \varepsilon$ is decomposed into an elastic $d \varepsilon^{e}$ and plastic part $d \varepsilon^{p}$ :

$\mathrm{d} \varepsilon=\mathrm{d} \varepsilon^{e}+\mathrm{d} \varepsilon^{p}$,

and the elastic predictor $\sigma_{i j}^{p r}$ is calculated as:

$\sigma_{i j}^{p r}=\sigma_{i j}^{0}+\lambda \delta_{i j} \Delta \varepsilon_{k k}^{e}+2 \mu \Delta \varepsilon_{i j}^{e}$,

where $\sigma_{i j}^{0}$ denotes the stress-state at instant $t$, and $\delta$ is the second-order identity tensor. $\mu$ and $\lambda$ are the shear modulus and Lamé constant which are defined as the functions of the Young's modulus $E$, and Poisson's ratio $v$ for isotropic materials as:

$\mu=\frac{E}{2(1+\nu)}, \lambda=\frac{\nu E}{(1-2 \nu)(1+\nu)}$.

The von Mises (equivalent) stress, $\bar{\sigma}$, based on purely elastic behavior (elastic predictor), can be calculated as:

$\bar{\sigma}=\sqrt{\frac{3}{2} S_{i j}^{p r} S_{i j}^{p r}}$,

where $S_{i j}^{p r}$ is the deviatoric stress and

$S_{i j}^{p r}=\sigma_{i j}^{p r}-\delta_{k k} \sigma_{m}$,

where $\sigma_{m}=\left(\sigma_{\mathrm{xx}}+\sigma_{\mathrm{yy}}+\sigma_{\mathrm{zz}}\right) / 3$ is the hydrostatic stress. Assuming isotropic plastic behavior, the von Mises yield function can be applied as:

$F(\sigma)=\bar{\sigma}-\sigma_{f}$,

where $\sigma_{f}$ is the flow stress calculated by the constitutive equation in Eq. (2), as:

$$
\sigma_{f}=\left[\sigma_{y}+B(\bar{\varepsilon})^{n}\right]\left[1+C \ln \left(\frac{\dot{\bar{\varepsilon}}}{\dot{\varepsilon}_{0}}\right)\right]\left[1-\left(\frac{T-T_{\mathrm{r}}}{T_{\mathrm{m}}-T_{\mathrm{r}}}\right)^{\mathrm{m}}\right] .
$$

The yield function (Eq. (30)) indicates that plastic deformation begins when the sum of the squares of the principal components of the deviatoric stress reaches a certain critical value. If the value of the yield function, $F(\sigma)$, is less than, or equal to, zero, the elastic predictor becomes the new state of stress at time $t+\Delta t$; Otherwise, the yield surface is extended and plastic flow occurs. The direction of plastic flow, $r$, is given by:

$r_{i j}=\frac{3}{2} \frac{S_{i j}^{p r}}{\bar{\sigma}}$.

The equivalent plastic strain rate can be calculated by:

$$
\dot{\bar{\varepsilon}}=\frac{\sqrt{2}}{3}\left(\left(\dot{e}_{x x}-\dot{e}_{y y}\right)^{2}+\left(\dot{e}_{y y}-\dot{e}_{z z}\right)^{2}+\left(\dot{e}_{z z}-\dot{e}_{x x}\right)^{2}+6\left(\dot{e}_{x y}^{2}+\dot{e}_{y z}^{2}+\dot{e}_{z x}^{2}\right)\right)^{1 / 2},
$$

where $\dot{e}_{i j}=\mathrm{de}_{i j} / \mathrm{d} t, d t$ is the current time step, and $d e_{i j}$ is the increment of deviatoric strain defined as:

$\mathrm{d} e_{i j}=\left[\begin{array}{ccc}\mathrm{d} \varepsilon_{x x}-\mathrm{d} \varepsilon_{\mathrm{m}} & \mathrm{d} \varepsilon_{x y} & \mathrm{~d} \varepsilon_{x z} \\ \mathrm{~d} \varepsilon_{y x} & \mathrm{~d} \varepsilon_{y y}-\mathrm{d} \varepsilon_{\mathrm{m}} & \mathrm{d} \varepsilon_{y z} \\ \mathrm{~d} \varepsilon_{z x} & \mathrm{~d} \varepsilon_{z y} & \mathrm{~d} \varepsilon_{z z}-\mathrm{d} \varepsilon_{\mathrm{m}}\end{array}\right]$,

where $\mathrm{d} \varepsilon_{m}=\left(\mathrm{d} \varepsilon_{\mathrm{xx}}+\mathrm{d} \varepsilon_{\mathrm{yy}}+\mathrm{d} \varepsilon_{\mathrm{zz}}\right) / 3$. The increment of equivalent plastic strain is approximately obtained by:

$\mathrm{d} \bar{\varepsilon}=\frac{\bar{\sigma}-\sigma_{f}}{3 \mu+h}$,

where $h$ stands for the hardening at the beginning of the increment which can be calculated by the tangent of the flow stress equation as:

$h=\frac{\mathrm{d} \sigma_{f}}{\mathrm{~d} \bar{\varepsilon}}$.

With respect to the implicit algorithm that we use with the Backward Euler integration method, the increment of the equivalent plastic strain at $t+\Delta t$ is determined by the information at $t+\Delta t$ whose value is unknown at time $t$, such that the iteration method needs to be applied to solve the incremental plastic consistency parameter, $\delta \bar{\varepsilon}$, until the convergence is satisfied,

$\delta \bar{\varepsilon}^{(n)}=\frac{\bar{\sigma}-3 \mu \mathrm{d} \bar{\varepsilon}^{(n)}-\sigma_{f}}{3 \mu+h^{(n)}}$,

$\mathrm{d} \bar{\varepsilon}^{(n+1)}=\mathrm{d} \bar{\varepsilon}^{(n)}+\delta \bar{\varepsilon}^{(n)}$,

where $n$ denotes the $n$th iteration. The equivalent plastic strain rate used in the iteration loops is obtained from:

$\dot{\bar{\varepsilon}}^{(n)}=\frac{\mathrm{d} \bar{\varepsilon}^{(n)}}{\mathrm{d} t}$.

At this point, the iteration is completed and the elastic-plastic solution is finalized. Then plastic strain, elastic strain, stress components and 
equivalent plastic strain are then updated as:

$\varepsilon_{i j}^{p(t+\Delta t)}=\varepsilon_{i j}^{p(t)}+r_{i j} \mathrm{~d} \bar{\varepsilon}$,

$\varepsilon_{i j}^{e(t+\Delta t)}=\varepsilon_{i j}^{e(t)}-r_{i j} \mathrm{~d} \bar{\varepsilon}$,

$\sigma_{i j}^{(t+\Delta t)}=\sigma_{i j}^{p r(t)}-2 G r_{i j} \mathrm{~d} \bar{\varepsilon}$,

$\bar{\varepsilon}^{(t+\Delta t)}=\bar{\varepsilon}^{(t)}+\mathrm{d} \bar{\varepsilon}$.

The dissipated inelastic energy, $W_{d}$ is also calculated as,

$W_{d}=\iiint_{V} \int_{t} \sigma_{f} \dot{\bar{\varepsilon}} \mathrm{d} V \mathrm{~d} t$

where $V$ is the geometrical domain occupied by the sample. The increment in plastic specific energy is defined as:

$\mathrm{d} W_{P}=\frac{1}{2} \frac{\sigma_{i j} \mathrm{~d} \varepsilon_{i j}}{\rho_{P}}$

The thermo-mechanical coupling is treated as local adiabatic heating with the temperature taken as an inner variable; the new temperature of the material $T^{\text {new }}$ is thus calculated using the temperature resulting from the plastic work, viz.:

$T^{\text {new }}=T^{\text {int }}+\frac{W_{d}}{\rho_{p} C_{\nu}}$,

where $T^{\text {int }}$ is the initial temperature, $\rho_{P}$ and $C_{v}$ are density and specific heat of the workpiece, respectively.

\section{DATA AVAILABILITY}

Experimental and numerical data from this study are available, respectively from Dr. Shan Cecilia Cao of the University of California Berkeley (email: shancao@berkeley. edu) and from Dr. Xiaochun Zhang of the Shanghai Institute of Applied Physics (email: zhangxiaochun@sinap.ac.cn) upon request.

\section{ACKNOWLEDGEMENTS}

The authors express their sincere gratitude to the National Key R\&D Program of China (Project No. 2017YFA0204403), the Major Program of National Natural Science Foundation of China: NSFC 51590892, the Shenzhen Science Technology and Innovation Commission under the Technology Research Grant no. JCYJ20160229165310679. They further thank the Research Center of AREVA NP for financial support, and to Mrs. E. Aublant, Dr. G. Kermouche and Prof. J.-M. Bergheau there for helpful discussions. J.L. acknowledges support from the Guangdong Provincial Department of Science and Technology under the Grant no. JSGG20141020103826038 and from the Shenzhen Science Technology and Innovation Commission under the Technology Research Grant no. 2014B050504003. R.O.R was supported by the Mechanical Behavior of Materials Program (K13) at the Lawrence Berkeley National Laboratory, funded by the U.S. Department of Energy, Office of Science, Office of Basic Energy Sciences, Materials Sciences and Engineering Division, under Contract No. DE-AC02-05CH11231. The authors also wish to thank Profs. C.T. Liu, T. F. Hung and Ming Dao for helpful discussions, and Yifan Zheng, Haokun Yang, Haojie Jiang, Yan Bao, Hui Yang and Fansheng Cheng for technical assistance.

\section{AUTHOR CONTRIBUTIONS}

S.C.C., X.Z., S-Q.S., J.L. and R.O.R. conceived the research; S.C.C. and X.Z., supervised by R.O.R., performed the numerical simulations and experiments, and analyzed the data with the help from S-Q.S., J.L. and R.O.R; S.C.C., X.Z. and R.O.R. wrote the manuscript, which all authors discussed and commented on.

\section{ADDITIONAL INFORMATION}

Competing interests: The authors declare no competing interests.

Publisher's note: Springer Nature remains neutral with regard to jurisdictional claims in published maps and institutional affiliations.

\section{REFERENCES}

1. Unal, O. \& Varol, R. Surface severe plastic deformation of AISI 304 via conventional shot peening, severe shot peening and repeening. Appl. Surf. Sci. 351, 289-295 (2015).

2. Majzoobi, G. H., Azizi, R. \& Alavi Nia, A. A three-dimensional simulation of shot peening process using multiple shot impacts. J. Mater. Process. Technol. 164, 1226-1234 (2005).

3. Cao, S. C. et al. Nature-inspired hierarchical steels. Sci. Rep. 8, 5088 (2018).

4. Cao, S. C. et al. Light-weight isometric-phase steels with superior strengthhardness-ductility combination. Scr. Mater. 154, 230-235 (2018).

5. Wu, G., Chan, K.-C., Zhu, L., Sun, L. \& Lu, J. Dual-phase nanostructuring as a route to high-strength magnesium alloys. Nature 545, 80 (2017).

6. Unal, O., Maleki, E. \& Varol, R. Effect of severe shot peening and ultra-low temperature plasma nitriding on Ti-6Al-4V alloy. Vacuum 150, 69-78 (2018).

7. Tao, N. R. et al. An investigation of surface nanocrystallization mechanism in iron induced by surface mechanical attrition treatment. Acta Mater. 50, 4603 (2002).

8. Sun, L., He, X. \& Lu, J. Nanotwinned and hierarchical nanotwinned metals: a review of experimental, computational and theoretical efforts. npj Computational. Materials 4, 6 (2018).

9. Xu, S., Xiong, L., Chen, Y. \& McDowell, D. L. Sequential slip transfer of mixedcharacter dislocations across $\Sigma 3$ coherent twin boundary in FCC metals: a concurrent atomistic-continuum study.npj Comput. Mater. 2, 15016 (2016).

10. Jung, J. et al. Continuum understanding of twin formation near grain boundaries of FCC metals with low stacking fault energy. npj Computational. Materials 3, 21 (2017).

11. Wegst, U. G. K., Bai, H., Saiz, E., Tomsia, A. P. \& Ritchie, R. O. Bioinspired structural materials. Nat. Mater. 14, 23-36 (2015).

12. Gludovatz, B. et al. A fracture-resistant high-entropy alloy for cryogenic applications. Science 345, 1153-1158 (2014).

13. Shekhawat, A. \& Ritchie, R. O. Toughness and strength of nanocrystalline graphene. Nat. Commun. 7, 10546 (2016).

14. Lu, K. \& Lu, J. Surface nanocrystallization (SNC) of metallic materials-presentation of the concept behind a new approach. J. Mater. Sci. \& Technol. 15, 193 (1999).

15. Ritchie, R. O. The conflicts between strength and toughness. Nat. Mater. 10, 817-822 (2011).

16. Sun, J. et al. Simultaneously improving surface mechanical properties and in vitro biocompatibility of pure titanium via surface mechanical attrition treatment combined with low-temperature plasma nitriding. Surf. Coat. Technol. 309, 382-389 (2017).

17. Guo, H.-Y. et al. Nanostructured laminar tungsten alloy with improved ductility by surface mechanical attrition treatment. Sci. Rep. 7, 1351 (2017).

18. Huang, L., Lu, J. \& Troyon, M. Nanomechanical properties of nanostructured titanium prepared by SMAT. Surf. \& Coat. Technol. 201, 208 (2006).

19. Lu, A. Q., Liu, G. \& Liu, C. M. Microstructural evolution of the surface layer of $316 \mathrm{~L}$ stainless steel induced by mechanical attrition. Acta Metall. Sin. 40, 943 (2004).

20. Wang, K., Tao, N. R., Liu, G., Lu, J. \& Lu, K. Plastic strain-induced grain refinement at the nanometer scale in copper. Acta Mater. 54, 5281 (2006).

21. Pei, Z. et al. Atomic structures of twin boundaries in hexagonal closepacked metallic crystals with particular focus on Mg. npj Comput. Mater. 3, 6 (2017).

22. Zhang, H. W., Hei, Z. K., Liu, G., Lu, J. \& Lu, K. Formation of nanostructured surface layer on AISI 304 stainless steel by means of surface mechanical attrition treatment. Acta Mater. 51, 1871 (2003).

23. Tao, N. R. et al. Mechanical and wear properties of nanostructured surface layer in iron induced by surface mechanical attrition treatment. J. Mater. Sci. \& Technol. 19, 563 (2003).

24. Shaw, L. L. \& Zhu, Y. T. in Materials Processing Handbook Ch. 31 (eds Groza, J. R., Shackelford, J. F., Lavernia, L. J. \& Powers M. I.) (CRC Press, Boca Raton, USA, 2007).

25. Alobaid, Y. F. Shot peening mechanics - experimental and theoretical-analysis. Mech. Mater. 19, 251 (1995).

26. Guagliano, M. Relating Almen intensity to residual stresses induced by shot peening: A numerical approach. J. Mater. Process. Technol. 110, 277 (2001).

27. Hertz, H. On the Contact of Elastic Solids. (Macmillan, Jones \& Schott, London, 1896).

28. Johnson, G. R. \& Cook, W. H. Fracture characteristics of three metals subjected to various strains, strain rates, temperatures and pressures. Eng. Fract. Mech. 21, 31 (1985).

29. Nasr, M. N. A., Ng, E. G. \& Elbestawi, M. A. Effects of strain hardening and initial yield strength on machining-induced residual stresses. J. Eng. Mater. \& Technol. 129, 567 (2007)

30. Chen, X. H., Lu, J., Lu, L. \& Lu, K. Tensile properties of a nanocrystalline $316 \mathrm{~L}$ austenitic stainless steel. Scr. Mater. 52, 1039 (2005). 
31. Dai, K., Villegas, J. \& Shaw, L. An analytical model of the surface roughness of an aluminum alloy treated with a surface nanocrystallization and hardening process. Scr. Mater. 52, 259-263 (2005).

32. Dai, K., Villegas, J., Stone, Z. \& Shaw, L. Finite element modeling of the surface roughness of $5052 \mathrm{Al}$ alloy subjected to a surface severe plastic deformation process. Acta Mater. 52, 5771-5782 (2004).

33. Moelans, N., Blanpain, B. \& Wollants, P. An introduction to phase-field modeling of microstructure evolution. Calphad-Comput. Coupling Phase Diagr. \& Thermochem. 32, 268 (2008).

34. Zimmermann, M., Schulze, V., Baron, H., Löhe, D., A novel 3D finite element simulation model for the prediction of the residual stress state after shot peening, (ed. Tosha, K.) Proceedings of the 10th International Conference on Shot Peening, 63-68 (Academy Common Meiji University, Tokyo Japan, 2008).

35. Klemenz, M., Schulze, V., Vöhringer, O. \& Löhe, D. Finite element simulation of the residual stress states after shot peening. Mater. Sci. Forum 524, (349-354 (2006).
Open Access This article is licensed under a Creative Commons Attribution 4.0 International License, which permits use, sharing, adaptation, distribution and reproduction in any medium or format, as long as you give appropriate credit to the original author(s) and the source, provide a link to the Creative Commons license, and indicate if changes were made. The images or other third party material in this article are included in the article's Creative Commons license, unless indicated otherwise in a credit line to the material. If material is not included in the article's Creative Commons license and your intended use is not permitted by statutory regulation or exceeds the permitted use, you will need to obtain permission directly from the copyright holder. To view a copy of this license, visit http://creativecommons. org/licenses/by/4.0/.

This is a U.S. government work and not under copyright protection in the U.S.; foreign copyright protection may apply 2019 